\title{
A technique of tripled coincidence points for solving a system of nonlinear integral equations in POCML spaces
}

\author{
Hasanen A. Hammad ${ }^{1 *}$ (D) and Manuel De La Sen²
}

\section{"Correspondence:}

h.elmagd89@gmail.com

'Department of Mathematics, Faculty of Science, Sohag University, Mohamed Farid-Elmeniawy, 82524 Sohag, Egypt

Full list of author information is available at the end of the article

\begin{abstract}
This manuscript aims to initiate some recent theoretical consequences related to tripled coincidence points for non-self mappings via the notion of $C$-type functions in partially ordered complete metric-like space (for short, POCML space). Our contributions unify and expand some previous studies in this line. Moreover, some corollaries and suitable examples are presented to demonstrate the novelty of the results established. Ultimately, two applications are given here to boost our theoretical consequences, the first one about the contributions of the integral type to obtain a triple coincidence points and the other application is about solving a system of nonlinear integral equations.
\end{abstract}

MSC: 46T99; 54H25; 47H10; 54E50

Keywords: Tripled coincidence point; Partially ordered set; Metric-like spaces; Nonlinear integral equations

\section{Introduction}

In the past, it was believed that scientific disciplines are completely separate; but now, after the tremendous development and modern theories in basic science techniques, they have become completely connected. For example, mathematics in which the level of development in different disciplines has varied dramatically in contemporary time. As an interesting example, fixed-point technologies offer a focal concept with many diverse usages. It has been and still is an important theoretical tool in many fields and various disciplines such as topology, game theory, optimal control, artificial intelligence, logic programming, dynamical systems (and chaos), functional analysis, differential equations, and economics. More clearly, for example, the technique of fixed point is applied for finding the solution of the equilibrium troubles in economics and game theory. In nonlinear integral equations, it is used to find analytical and numerical solutions to Fredholm integral equations [1-5], etc.

The ideas of mixed-monotone functions and coupled fixed point were initiated in the paper [6]. Under these ideas, some main results in partially ordered metric spaces have been driven by the authors [6]. For enjoyable specifics on coupled fixed point consequences and related topics in abstract spaces, the reader can refer to [7-27].

(c) The Author(s) 2020. This article is licensed under a Creative Commons Attribution 4.0 International License, which permits use, sharing, adaptation, distribution and reproduction in any medium or format, as long as you give appropriate credit to the original author(s) and the source, provide a link to the Creative Commons licence, and indicate if changes were made. The images or other third party material in this article are included in the article's Creative Commons licence, unless indicated otherwise in a credit line to the material. If material is not included in the article's Creative Commons licence and your intended use is not permitted by statutory regulation or exceeds the permitted use, you will need to obtain permission directly from the copyright holder. To view a copy of this licence, visit http://creativecommons.org/licenses/by/4.0/. 
Pivotal results related to a triple fixed point (established in 2011 by Berinde and Borcut [28]) were presented in partially ordered metric spaces. For more topics of this notion, we cite papers [29-35].

Definition 1.1 ([28]) It is said that a trio $(\wp, \hbar, ð) \in \chi^{3}$ is a tripled fixed point of a selfmapping $\Re: \chi^{3} \rightarrow \chi$ if $\wp=\Re(\wp, \hbar, ð), \hbar=\Re(\hbar, \wp, \hbar)$, and $ð=\Re(ð, \hbar, \wp)$.

Definition $1.2([29])$ A trio $(\wp, \hbar, ð) \in \chi^{3}$ on a nonempty set $\chi$ is called a tripled coincidence point of the two self-mappings $\Re: \chi^{3} \rightarrow \chi$ and $\Theta: \chi \rightarrow \chi$ if $\Im \wp=\Re(\wp, \hbar, ð)$, $\Im \hbar=\Re(\hbar, \wp, \hbar)$, and $\Im \partial=\Re(ð, \hbar, \wp)$.

Definition 1.3 ([29]) Consider that $\chi \neq \varnothing$ is a set, a trio $(\wp, \hbar, \check{\partial}) \in \chi^{3}$ is said to be a tripled common fixed point of $\Re: \chi \rightarrow \chi$ and $\Theta: \chi \rightarrow \chi$ if $\wp=\Im \wp=\Re(\wp, \hbar, ठ)$, $\hbar=\Im \hbar=\Re(\hbar, \wp, \hbar)$, and $\partial=\Im ð=\Re(ð, \hbar, \wp)$.

Definition 1.4([31]) Suppose that $\chi \neq \emptyset$ is a set, the mappings $\Re: \chi^{3} \rightarrow \chi$ and $\Theta: \chi \rightarrow \chi$ are commutative $\Im(\Re(\wp, \hbar, \partial))=\Re(\Im \wp, \Im \wp, \Im \wp)$ for all $\wp, \hbar, ð \in \chi$.

Definition 1.5 ([28]) A mapping $\Re: \chi^{3} \rightarrow \chi$ on a partially ordered set $(\chi, \precsim)$ has a mixedmonotone property if, for any $\wp, \hbar, \partial \in \chi$,

$$
\begin{aligned}
& \wp_{1}, \wp_{2} \in \chi, \wp_{1} \precsim \wp_{2} \text { implies } \quad \Re\left(\wp_{1}, \hbar, ð\right) \precsim \Re\left(\wp_{2}, \hbar, ð\right) \text {, } \\
& \hbar_{1}, \hbar_{2} \in \chi, \hbar_{1} \precsim \hbar_{2} \text { implies } \quad \Re\left(\wp, \hbar_{1}, \partial\right) \succsim \Re\left(\wp, \hbar_{2}, \partial\right) \text {, } \\
& \partial_{1}, \partial_{2} \in \chi, \partial_{1} \precsim \partial_{2} \text { implies } \Re\left(\wp, \hbar, \partial_{1}\right) \precsim \Re\left(\wp, \hbar, \partial_{2}\right) \text {. }
\end{aligned}
$$

Recently, Aydi et al. extended the property of mixed-monotone to $\mathfrak{\Im}$-mixed-monotone as follows.

Definition 1.6 ([36]) A mapping $\Re: \chi^{3} \rightarrow \chi$ on a partially ordered set $(\chi, \precsim)$ has a mixed $\Im$-monotone property where $\mathfrak{\Im}: \chi \rightarrow \chi$ if, for any $\wp, \hbar, \precsim \in \chi$,

$$
\begin{aligned}
& \wp_{1}, \wp_{2} \in \chi, \Im \wp_{1} \precsim \Im \wp_{2} \text { implies } \quad \mathfrak{N}\left(\wp_{1}, \hbar, \partial\right) \precsim \Re\left(\wp_{2}, \hbar, ð\right) \text {, } \\
& \hbar_{1}, \hbar_{2} \in \chi, \Im \hbar_{1} \precsim \Im \hbar_{2} \text { implies } \Re\left(\wp, \hbar_{1}, \partial\right) \succsim \Re\left(\wp, \hbar_{2}, \partial\right) \text {, } \\
& \check{\partial}_{1}, \check{\partial}_{2} \in \chi, \Im \check{\partial}_{1} \precsim \Im \widetilde{J}_{2} \text { implies } \quad \Re\left(\wp, \hbar, \check{\partial}_{1}\right) \precsim \Re\left(\wp, \hbar, \check{\partial}_{2}\right) \text {. }
\end{aligned}
$$

The first theorem concerned with a tripled fixed point of the mapping which has a mixed-monotone property in a partially ordered set was introduced as follows.

Theorem 1.7 ([28]) Let $(\chi, \precsim, \xi)$ be a POCM space. Consider the mapping $\Re: \chi^{3} \rightarrow \chi$ such that:

(i) $\Re$ has a mixed-monotone property;

(ii) Either $\Re$ is continuous or $\chi$ has the following properties:

(a) $l_{n} \precsim l$ if a nondecreasing sequence $l_{n} \rightarrow l$ for all $n$,

(b) $j_{n} \succsim j$ if a nonincreasing sequence $j_{n} \rightarrow j$ for all $n$; 
(iii) There are $\alpha, \beta, \gamma \geq 0$ with $\alpha+\beta+\gamma<1$ such that

$$
\xi(\Re(\wp, \hbar, ð), \Re(x, y, z)) \leq \alpha \xi(\wp, x)+\beta \xi(\hbar, y)+\gamma \xi(c, z)
$$

for any $\wp, \hbar, c, x, y, z \in \chi$, for which $\wp \precsim x, y \precsim \hbar$, and $\precsim \precsim z$. If there exist $\wp_{\circ}, \hbar_{\circ}, \partial_{\circ} \in \chi$ such that $\wp_{\circ} \precsim \Re\left(\wp_{\circ}, \hbar_{\circ}, \partial_{\circ}\right), \hbar_{\circ} \succsim \Re\left(\hbar_{\circ}, \wp_{\circ}, \hbar_{\circ}\right)$, and $\partial_{\circ} \precsim \Re\left(\varpi_{\circ}, \hbar_{\circ}, \wp_{\circ}\right)$. Then $\Re$ has a tripled fixed point.

\section{Metric-like spaces and C-class functions}

In 1994, the notion of spaces with the same nonzero distance from the points was shown by Matthews [37]. The authors [38] improved it in dislocated and dislocated quasi-metric spaces. In [39] the concept of a metric-like space was discussed, which is an important extension of the spaces defined in $[38,39]$.

Very recently, many fixed point results on metric-like spaces have been provided. For more specifics, see [40-49].

Now, we state some basic significance of metric-like spaces.

Definition 2.1 ([39]) A mapping $\xi: \chi \times \chi \rightarrow[0,+\infty)$ on a nonempty set $\chi$ is called metric-like if, for all $\wp, \hbar, \partial \in \chi$, the following assumptions hold:

$\left(\xi_{1}\right) \xi(\wp, \hbar)=0$ implies $\wp=\hbar$;

$\left(\xi_{2}\right) \xi(\wp, \hbar)=\xi(\hbar, \wp)$;

$\left(\xi_{3}\right) \xi(\wp, ð) \leq \xi(\wp, \hbar)+\xi(\hbar, \precsim)$.

Thus, the parenthesis $(\chi, \xi)$ is called a metric-like space.

For $\wp \in \chi, \xi(\wp, \wp)$ may be positive except that a metric-like mapping satisfies all the assumptions of ordinary metric.

Definition 2.2 ([39]) Let a sequence of points $\left\{\wp_{n}\right\}$ be on $\chi$ in a metric-like space $(\chi, \xi)$. A point $\wp \in \chi$ is called the limit of the sequence $\left\{\wp_{n}\right\}$ if $\lim _{n \rightarrow \infty} \xi\left(\wp, \wp_{n}\right)=\xi(\wp, \wp)$, and we say that the point $\wp$ is a limit point of the sequence $\left\{\wp_{n}\right\}$.

Definition 2.3 ([39]) Assume that $(\chi, \xi)$ is a metric-like space.

(i) A sequence $\left\{\wp_{n}\right\}$ is called a $\xi$-Cauchy sequence if $\lim _{m, n \rightarrow+\infty} \xi\left(\wp_{m}, \wp_{n}\right)$ exists and is finite;

(ii) If every $\xi$-Cauchy sequence $\left\{\wp_{n}\right\}$ in $\chi$ converges to $\wp \in \chi$, with $\lim _{m, n \rightarrow+\infty} \xi\left(\wp_{m}, \wp_{n}\right)=\omega(\wp, \wp)=\lim _{n \rightarrow+\infty} \xi\left(\wp_{n}, \wp\right)$, then the space $(\chi, \xi)$ is called complete.

Lemma 2.4 ([40]) Consider $(\chi, \xi)$ to be a metric-like space and $\left\{\wp_{n}\right\}$ be a sequence of $\chi$ such that $a_{n} \rightarrow a$ as $n \rightarrow+\infty$ and $\xi(\wp, \wp)=0$. Then $\lim _{n \rightarrow+\infty} \xi\left(\wp_{n}, \hbar\right)=\xi(\wp, \hbar)$ for all $\hbar \in \chi$.

Lemma 2.5 ([40]) Suppose that $(\chi, \xi)$ is a metric-like space. Then

(i) if $\xi(\wp, \hbar)=0$, then $\xi(\wp, \wp)=\xi(\hbar, \hbar)=0$;

(ii) if $\left\{\wp_{n}\right\}$ is a sequence such that $\lim _{n \rightarrow+\infty} \xi\left(\wp_{n}, \wp_{n+1}\right)=0$, then

$$
\lim _{n \rightarrow+\infty} \xi\left(\wp_{n}, \wp_{n}\right)=\lim _{n \rightarrow+\infty} \xi\left(\wp_{n+1}, \wp_{n+1}\right)=0
$$


(iii) if $\wp \neq \hbar$, then $\xi(\wp, \hbar)>0$;

(iv) $\xi(\wp, \wp) \leq \frac{2}{n} \sum_{i=1}^{n} \xi\left(\wp, \wp_{i}\right)$ holds for all $\wp, \wp_{i} \in \chi$, where $1 \leq i \leq n$.

Here, we assume that $\Pi=\{\pi:[0,+\infty) \rightarrow[0,+\infty)$ is a nondecreasing function and lower semi-continuous such that $\pi(v)=0 \Leftrightarrow v=0$.

In 2014, the idea of $C$-type functions which cover a large class of contractive conditions was presented by Ansari [50] as follows.

Definition 2.6 ([50]) A mapping $\Lambda:[0,+\infty)^{2} \rightarrow \mathbb{R}$ is called $C$-type function if it is continuous and fulfills the following hypotheses:

(1) $\Lambda(\lambda, \mu) \leq \lambda$;

(2) $\Lambda(\lambda, \mu)=\lambda$ implies that either $\lambda=0$ or $\mu=0$ for all $\lambda, \mu \in[0, \infty)$.

We symbolize the $C$-type functions as $\complement$.

Example 2.7 For all $\lambda, \mu \in[0, \infty)$, the following functions $\Re:[0, \infty)^{2} \rightarrow \mathbb{R}$ are elements of $\complement$ :

- $\Lambda(\lambda, \mu)=\lambda-\mu$;

- $\Lambda(\lambda, \mu)=\varsigma \lambda, 0<\varsigma<1$

- $\Lambda(\lambda, \mu)=\frac{\lambda}{(1+\mu)^{\varepsilon}} ; \varepsilon \in(0,+\infty)$;

- $\Lambda(\lambda, \mu)=\frac{\log \left(\mu+c^{\lambda}\right)}{(1+\mu)}, c>1$;

- $\Lambda(\lambda, 1)=\frac{\ln \left(1+d^{\lambda}\right)}{2}, d>e$;

- $\Lambda(\lambda, \mu)=\theta(\mu)$, where $\theta:[0,+\infty) \rightarrow[0,+\infty)$ is an upper semi-continuous function such that $\theta(0)=0$, and $\theta(\mu)<\mu$ for $\mu>0$;

- $\Lambda(\lambda, \mu)=\lambda \Omega(\lambda), \Omega:[0,1) \rightarrow[0,1)$

- $\Lambda(\lambda, \mu)=\lambda-\theta(\lambda)$

- $\Lambda(\lambda, \mu)=\lambda-\frac{\mu}{1-\mu}$

- $\Lambda(\lambda, \mu)=\lambda q(\lambda, \mu)$, where $q:[0,1) \times[0,1) \rightarrow[0,1)$ is a continuous function such that for all $\lambda, \mu>0, q(\lambda, \mu)<1$.

In this article, some new tripled coincidence point consequences for mixed-monotone mappings via the notion of $C$-type functions in POCbML spaces are introduced. Some examples to back our work are showed. Also, some theoretical results under various contractive conditions are discussed as corollaries. Eventually, some important results in integral types and the existence of solutions of a system of nonlinear integral equations are presented here as applications.

\section{Main theorems}

Theorem 3.1 Assume that $\Re: \chi^{3} \rightarrow \chi$ and $\Theta: \chi \rightarrow \chi$ are two mappings on a POCML space $(\chi, \precsim, \xi)$ such that:

(i) $\mathfrak{R}\left(\chi^{3}\right) \subseteq \Im(\chi)$;

(ii) $\Re$ is continuous;

(iii) $\Im$ is continuous and commutes with $\Re$;

(iv) $\Re$ has a mixed $\Im$-monotone property; 
(v) there are $\pi \in \Pi, \zeta \geq 0$, and $\Lambda \in \complement$ such that

$$
\begin{aligned}
\xi(\Re(\wp, \hbar, \Im), \Re(x, y, z)) \\
\quad \leq \Lambda\left(\begin{array}{c}
\pi(\max \{\xi(\Im(\wp), \Im(x)), \xi(\Im(\hbar), \Im(y)), \xi(\Im(ð), \Im(z))\}), \\
\zeta \max \{\xi(\Im(\wp), \Im(x)), \xi(\Im(\hbar), \Im(y)), \xi(\Im(\Im), \Im(z))\}
\end{array}\right)
\end{aligned}
$$

for any $\wp, \hbar, \precsim, x, y, z \in \chi$, for which $\Im(\wp) \precsim \Im(x), \Im(y) \precsim \Im(\hbar)$, and $\Im(ð) \precsim \Im(z)$. If there exist $\wp_{\circ}, \hbar_{\circ}, \partial_{\circ} \in \chi$ such that $\Im\left(\wp_{\circ}\right) \precsim \Re\left(\wp_{\circ}, \hbar_{\circ}, \partial_{\circ}\right), \Im\left(\hbar_{\circ}\right) \succsim \Re\left(\hbar_{\circ}, \wp_{\circ}, \partial_{\circ}\right)$, and $\Im\left(\varpi_{\circ}\right) \precsim$ $\Re\left(\Im_{\circ}, \hbar_{\circ}, \wp_{\circ}\right)$, then $\Re$ and $\Im$ have a tripled coincidence point.

Proof Let $\wp_{\circ}, \hbar_{\circ}, \partial_{\circ} \in \chi$ with $\Im\left(\wp_{\circ}\right) \precsim \Re\left(\wp_{\circ}, \hbar_{\circ}, \partial_{\circ}\right), \Im\left(\hbar_{\circ}\right) \succsim \Re\left(\hbar_{\circ}, \wp_{\circ}, \partial_{\circ}\right)$, and $\Im\left(\partial_{\circ}\right) \precsim$ $\Re\left(\check{\partial}_{\circ}, \hbar_{\circ}, \wp_{\circ}\right)$. Since $\Re\left(\chi^{3}\right) \subseteq \Im(\chi)$, there exist $\wp_{1}, \hbar_{1}, \check{\partial}_{1} \in \chi$ such that

$$
\Im\left(\wp_{1}\right)=\Re\left(\wp_{\circ}, \hbar_{\circ}, \partial_{\circ}\right), \quad \Im\left(\hbar_{1}\right)=\Re\left(\hbar_{\circ}, \wp_{\circ}, \partial_{\circ}\right), \quad \text { and } \quad \Im\left(\partial_{1}\right)=\Re\left(\varpi_{\circ}, \hbar_{\circ}, \wp_{\circ}\right) \text {. }
$$

Continuing with the same scenario, there are $\left\{\wp_{n}\right\},\left\{\hbar_{n}\right\}$, and $\left\{\partial_{n}\right\}$ in $\chi$ such that

$$
\begin{aligned}
& \Im\left(\wp_{n+1}\right)=\Re\left(\wp_{n}, \hbar_{n}, ð_{n}\right), \quad \Im\left(\hbar_{n+1}\right)=\Re\left(\hbar_{n}, \wp_{n}, ð_{n}\right), \quad \text { and } \\
& \Im\left(\bigodot_{n+1}\right)=\Re\left(\Im_{n}, \hbar_{n}, \wp_{n}\right) .
\end{aligned}
$$

By induction, we shall show that

$$
\begin{aligned}
& \Im\left(\wp_{n}\right) \precsim \Im\left(\wp_{n+1}\right), \quad \Im\left(\hbar_{n+1}\right) \precsim \Im\left(\hbar_{n}\right), \quad \text { and } \\
& \Im\left(( _ { n } ) \precsim \Im \left(\left(_{n+1}\right) \quad \text { for all } n \in \mathbb{N} \cup\{0\} .\right.\right.
\end{aligned}
$$

Since $\Im\left(\wp_{\circ}\right) \precsim \Re\left(\wp_{\circ}, \hbar_{\circ}, \partial_{\circ}\right), \Im\left(\hbar_{\circ}\right) \succsim \Re\left(\hbar_{\circ}, \wp_{\circ}, \check{\partial}_{\circ}\right)$, and $\Im\left(\varpi_{\circ}\right) \precsim \Re\left(\varpi_{\circ}, \hbar_{\circ}, \wp_{\circ}\right)$, and by $(2)$, we have

$$
\Im\left(\wp_{\circ}\right) \precsim \Im\left(\wp_{1}\right), \quad \Im\left(\hbar_{\circ}\right) \succsim \Im\left(\hbar_{1}\right), \quad \text { and } \quad \Im\left(\oiint_{\circ}\right) \precsim \Im\left(\oiint_{1}\right)
$$

This leads to (4) fulfilled for $n=0$. Consider (4) to be realized for some fixed $n \in \mathbb{N}$. Because $\Re$ has a mixed $\Im$-monotone property, we have

$$
\begin{aligned}
& \Im\left(\wp_{n+1}\right)=\Re\left(\wp_{n}, \hbar_{n}, \partial_{n}\right) \precsim \Re\left(\wp_{n+1}, \hbar_{n}, \partial_{n}\right) \precsim \Re\left(\wp_{n+1}, \hbar_{n}, \partial_{n+1}\right) \\
& \precsim \Re\left(\wp_{n+1}, \hbar_{n+1}, \partial_{n+1}\right)=\Im\left(\wp_{n+2}\right), \\
& \Im\left(\hbar_{n+2}\right)=\Re\left(\hbar_{n+1}, \wp_{n+1}, \hbar_{n+1}\right) \precsim \Re\left(\hbar_{n+1}, \wp_{n}, \hbar_{n+1}\right) \precsim \Re\left(\hbar_{n}, \wp_{n}, \hbar_{n+1}\right) \\
& \precsim \Re\left(\hbar_{n}, \wp_{n}, \hbar_{n}\right)=\Im\left(\hbar_{n+1}\right),
\end{aligned}
$$

and

$$
\begin{aligned}
\Im\left(\coprod_{n+1}\right) & =\Re\left(\coprod_{n}, \hbar_{n}, \wp_{n}\right) \precsim \Re\left(\coprod_{n+1}, \hbar_{n}, \wp_{n}\right) \precsim \Re\left(\coprod_{n+1}, \hbar_{n+1}, \wp_{n}\right) \\
& \precsim \Re\left(\coprod_{n+1}, \hbar_{n+1}, \wp_{n+1}\right)=\Im\left(\coprod_{n+2}\right) .
\end{aligned}
$$


Thus, (4) is fulfilled. For each $n \in \mathbb{N}$, suppose that

$$
\Im\left(\wp_{n}\right)=\Im\left(\wp_{n+1}\right), \quad \Im\left(\hbar_{n}\right)=\Im\left(\hbar_{n+1}\right), \quad \text { and } \quad \Im\left(\Im_{n}\right)=\Im\left(\Im_{n+1}\right)
$$

By (3), we deduce that a trio $\left(\wp_{n}, \hbar_{n}, \partial_{n}\right)$ is a coincidence point of $\Re$ and $\Im$. Now, consider at least for any $n \in \mathbb{N}$

$$
\Im\left(\wp_{n}\right) \neq \Im\left(\wp_{n+1}\right), \quad \Im\left(\hbar_{n}\right) \neq \Im\left(\hbar_{n+1}\right), \quad \text { and } \quad \Im\left(\Im_{n}\right) \neq \Im\left(\Im_{n+1}\right) \text {. }
$$

Applying (1), and since $\pi$ is nondecreasing, we can get

$$
\begin{aligned}
& \xi\left(\Im\left(\wp_{n}\right), \Im\left(\wp_{n+1}\right)\right) \\
& =\xi\left(\Re\left(\wp_{n-1}, \hbar_{n-1}, \ddot{\partial}_{n-1}\right), \Re\left(\wp_{n}, \hbar_{n}, \ddot{\partial}_{n}\right)\right) \\
& \leq \Lambda\left(\begin{array}{c}
\pi\left(\max \left\{\xi\left(\Im\left(\wp_{n-1}\right), \Im\left(\wp_{n}\right)\right), \xi\left(\Im\left(\hbar_{n-1}\right), \Im\left(\hbar_{n}\right)\right), \xi\left(\Im\left(\Im_{n-1}\right), \Im\left(\Im_{n}\right)\right)\right\}\right), \\
\zeta \max \left\{\xi\left(\Im\left(\wp_{n-1}\right), \Im\left(\wp_{n}\right)\right), \xi\left(\Im\left(\hbar_{n-1}\right), \Im\left(\hbar_{n}\right)\right), \xi\left(\Im\left(\varpi_{n-1}\right), \Im\left(\Im_{n}\right)\right)\right\}
\end{array}\right) \\
& \leq \pi\left(\max \left\{\xi\left(\Im\left(\wp_{n-1}\right), \Im\left(\wp_{n}\right)\right), \xi\left(\Im\left(\hbar_{n-1}\right), \Im\left(\hbar_{n}\right)\right), \xi\left(\Im\left(\Im_{n-1}\right), \Im\left(\partial_{n}\right)\right)\right\}\right), \\
& \xi\left(\Im\left(\hbar_{n+1}\right), \Im\left(\hbar_{n}\right)\right) \\
& =\xi\left(\Re\left(\hbar_{n}, \wp_{n}, \hbar_{n}\right), \Re\left(\hbar_{n-1}, \wp_{n-1}, \hbar_{n-1}\right)\right) \\
& \leq \Lambda\left(\begin{array}{c}
\pi\left(\max \left\{\xi\left(\Im\left(\hbar_{n-1}\right), \Im\left(\hbar_{n}\right)\right), \xi\left(\Im\left(\wp_{n-1}\right), \Im\left(\wp_{n}\right)\right), \xi\left(\Im\left(\hbar_{n-1}\right), \Im\left(\hbar_{n}\right)\right)\right\}\right), \\
\zeta \max \left\{\xi\left(\Im\left(\hbar_{n-1}\right), \Im\left(\hbar_{n}\right)\right), \xi\left(\Im\left(\wp_{n-1}\right), \Im\left(\wp_{n}\right)\right), \xi\left(\Im\left(\hbar_{n-1}\right), \Im\left(\hbar_{n}\right)\right)\right\}
\end{array}\right) \\
& =\Lambda\left(\begin{array}{c}
\pi\left(\max \left\{\xi\left(\Im\left(\hbar_{n-1}\right), \Im\left(\hbar_{n}\right)\right), \xi\left(\Im\left(\wp_{n-1}\right), \Im\left(\wp_{n}\right)\right)\right\}\right), \\
\zeta \max \left\{\xi\left(\Im\left(\hbar_{n-1}\right), \Im\left(\hbar_{n}\right)\right), \xi\left(\Im\left(\wp_{n-1}\right), \Im\left(\wp_{n}\right)\right)\right\}
\end{array}\right) \\
& \leq \pi\left(\max \left\{\xi\left(\Im\left(\hbar_{n-1}\right), \Im\left(\hbar_{n}\right)\right), \xi\left(\Im\left(\wp_{n-1}\right), \Im\left(\wp_{n}\right)\right)\right\}\right),
\end{aligned}
$$

and

$$
\begin{aligned}
& \xi\left(\Im\left(\coprod_{n}\right), \Im\left(\Im_{n+1}\right)\right) \\
& =\xi\left(\Xi\left(\Im_{n-1}, \hbar_{n-1}, a_{n-1}\right), \Xi\left(\varlimsup_{n}, \hbar_{n}, a_{n}\right)\right) \\
& \leq \Lambda\left(\begin{array}{c}
\pi\left(\max \left\{\xi\left(\Im\left(\Im_{n-1}\right), \Im\left(\Im_{n}\right)\right), \xi\left(\Im\left(\hbar_{n-1}\right), \Im\left(\hbar_{n}\right)\right), \xi\left(\Im\left(\wp_{n-1}\right), \Im\left(\wp_{n}\right)\right)\right\}\right), \\
\zeta \max \left\{\xi\left(\Im\left(\Im_{n-1}\right), \Im\left(\varpi_{n}\right)\right), \xi\left(\Im\left(\hbar_{n-1}\right), \Im\left(\hbar_{n}\right)\right), \xi\left(\Im\left(\wp_{n-1}\right), \Im\left(\wp_{n}\right)\right)\right\}
\end{array}\right) \\
& \leq \pi\left(\max \left\{\xi\left(\Im\left(\Im_{n-1}\right), \Im\left(\left(_{n}\right)\right), \xi\left(\Im\left(\hbar_{n-1}\right), \Im\left(\hbar_{n}\right)\right), \xi\left(\Im\left(\wp_{n-1}\right), \Im\left(\wp_{n}\right)\right)\right\}\right) .\right.
\end{aligned}
$$

Take into account that $\pi(v)<v$ for all $v>0$, then by (5), (6), and (7), one can get

$$
\begin{aligned}
& 0<\max \left\{\xi\left(\Im\left(\wp_{n}\right), \Im\left(\wp_{n+1}\right)\right), \xi\left(\Im\left(\hbar_{n+1}\right), \Im\left(\hbar_{n}\right)\right), \xi\left(\Im\left(\Im_{n}\right), \Im\left(\Im_{n+1}\right)\right)\right\} \\
& \leq \pi\left(\max \left\{\xi\left(\Im\left(\Im_{n-1}\right), \Im\left(\varpi_{n}\right)\right), \xi\left(\Im\left(\hbar_{n-1}\right), \Im\left(\hbar_{n}\right)\right), \xi\left(\Im\left(\wp_{n-1}\right), \Im\left(\wp_{n}\right)\right)\right\}\right) \\
& <\max \left\{\xi\left(\Im\left(\Im_{n-1}\right), \Im\left(\Im_{n}\right)\right), \xi\left(\Im\left(\hbar_{n-1}\right), \Im\left(\hbar_{n}\right)\right), \xi\left(\Im\left(\wp_{n-1}\right), \Im\left(\wp_{n}\right)\right)\right\} .
\end{aligned}
$$


It follows by (8) that

$$
\begin{aligned}
\Delta_{n} & =\max \left\{\xi\left(\Im\left(\wp_{n}\right), \Im\left(\wp_{n+1}\right)\right), \xi\left(\Im\left(\hbar_{n+1}\right), \Im\left(\hbar_{n}\right)\right), \xi\left(\Im\left(\varpi_{n}\right), \Im\left(\varpi_{n+1}\right)\right)\right\} \\
& <\max \left\{\xi\left(\Im\left(\varpi_{n-1}\right), \Im\left(\varpi_{n}\right)\right), \xi\left(\Im\left(\hbar_{n-1}\right), \Im\left(\hbar_{n}\right)\right), \xi\left(\Im\left(\wp_{n-1}\right), \Im\left(\wp_{n}\right)\right)\right\} .
\end{aligned}
$$

Thus, $\Delta_{n}$ is a positive decreasing sequence. So there is $\kappa \geq 0$ such that

$$
\lim _{n \rightarrow+\infty} \Delta_{n}=\kappa
$$

Consider $\kappa>0$ and $n \rightarrow+\infty$ in (8), we can write

$$
\begin{aligned}
\kappa & \leq \lim _{n \rightarrow+\infty} \pi\left(\max \left\{\xi\left(\Im\left(\varpi_{n-1}\right), \Im\left(\varpi_{n}\right)\right), \xi\left(\Im\left(\hbar_{n-1}\right), \Im\left(\hbar_{n}\right)\right), \xi\left(\Im\left(\wp_{n-1}\right), \Im\left(\wp_{n}\right)\right)\right\}\right) \\
& <\lim _{n \rightarrow+\infty} \Delta_{n-1}=\kappa .
\end{aligned}
$$

This is an inconsistency, hence

$$
\lim _{n \rightarrow+\infty} \Delta_{n}=0
$$

Now, we shall demonstrate that $\left\{\Im\left(\wp_{n}\right)\right\}$, $\left\{\mathfrak{\Im}\left(\hbar_{n}\right)\right\}$, and $\left\{\Im\left(\Im_{n}\right)\right\}$ are Cauchy sequences by an inconsistency method. So, let one of them not be Cauchy, that is,

$$
\begin{aligned}
& \lim _{n, m \rightarrow+\infty} \xi\left(\Im\left(\wp_{n}\right), \Im\left(\wp_{m}\right)\right) \neq 0, \quad \text { or } \quad \lim _{n, m \rightarrow+\infty} \xi\left(\Im\left(\hbar_{n}\right), \Im\left(\hbar_{m}\right)\right) \neq 0, \quad \text { or } \\
& \lim _{n, m \rightarrow+\infty} \xi\left(\Im\left(\Im_{n}\right), \Im\left(\oiint_{m}\right)\right) \neq 0 .
\end{aligned}
$$

In other words, there are $\epsilon>0$ and integers subsequences $\left\{m_{k}\right\}$ and $\left\{n_{k}\right\}$ with $n_{k}>m_{k}>k$ such that

$$
\max \left\{\xi\left(\Im\left(\wp_{m_{k}}\right), \Im\left(\wp_{n_{k}}\right)\right), \xi\left(\Im\left(\hbar_{m_{k}}\right), \Im\left(\hbar_{n_{k}}\right)\right), \xi\left(\Im\left(\varpi_{m_{k}}\right), \Im\left(\varpi_{n_{k}}\right)\right)\right\} \geq \epsilon
$$

If we adopt $m_{k}$ the little integer with $n_{k}>m_{k}$ satisfying (10), then the following connection holds:

$$
\max \left\{\xi\left(\Im\left(\wp_{m_{k}}\right), \Im\left(\wp_{n_{k}}\right)\right), \xi\left(\Im\left(\hbar_{m_{k}}\right), \Im\left(\hbar_{n_{k}}\right)\right), \xi\left(\Im\left(\varpi_{m_{k}}\right), \Im\left(\varpi_{n_{k}}\right)\right)\right\}<\epsilon .
$$

Thus, by (9), stipulation $\left(\xi_{3}\right)$, and (11), we have

$$
\begin{aligned}
\lim _{k \rightarrow+\infty} \xi\left(\Im\left(\wp_{m_{k}}\right), \Im\left(\wp_{n_{k}}\right)\right) & \leq \lim _{k \rightarrow+\infty}\left(\xi\left(\Im\left(\wp_{m_{k}}\right), \Im\left(\wp_{n_{k}-1}\right)\right)+\xi\left(\Im\left(\wp_{n_{k}-1}\right), \Im\left(\wp_{n_{k}}\right)\right)\right) \\
& \leq \lim _{k \rightarrow+\infty} \xi\left(\Im\left(\wp_{m_{k}}\right), \Im\left(\wp_{n_{k}-1}\right)\right) \leq \epsilon .
\end{aligned}
$$

By the same logic, we can get

$$
\begin{aligned}
& \lim _{k \rightarrow+\infty} \xi\left(\Im\left(\hbar_{m_{k}}\right), \Im\left(\hbar_{n_{k}}\right)\right) \leq \lim _{k \rightarrow+\infty} \xi\left(\Im\left(\hbar_{m_{k}}\right), \Im\left(\hbar_{n_{k}-1}\right)\right) \leq \epsilon, \\
& \lim _{k \rightarrow+\infty} \xi\left(\Im\left(\Im_{m_{k}}\right), \Im\left(\varlimsup_{n_{k}}\right)\right) \leq \lim _{k \rightarrow+\infty} \xi\left(\Im\left(\varpi_{m_{k}}\right), \Im\left(\varpi_{n_{k}-1}\right)\right) \leq \epsilon .
\end{aligned}
$$


Once more, by (11), we can note

$$
\begin{aligned}
\xi\left(\Im\left(\wp_{m_{k}}\right), \Im\left(\wp_{n_{k}}\right)\right) \leq & \xi\left(\Im\left(\wp_{m_{k}}\right), \Im\left(\wp_{m_{k}-1}\right)\right) \\
& +\xi\left(\Im\left(\wp_{m_{k}-1}\right), \Im\left(\wp_{n_{k}-1}\right)\right)+\xi\left(\Im\left(\wp_{n_{k}-1}\right), \Im\left(\wp_{n_{k}}\right)\right) \\
\leq & \xi\left(\Im\left(\wp_{m_{k}}\right), \Im\left(\wp_{m_{k}-1}\right)\right)+\xi\left(\Im\left(\wp_{m_{k}-1}\right), \Im\left(\wp_{m_{k}}\right)\right) \\
& +\xi\left(\Im\left(\wp_{m_{k}}\right), \Im\left(\wp_{n_{k}-1}\right)\right)+\xi\left(\Im\left(\wp_{n_{k}-1}\right), \Im\left(\wp_{n_{k}}\right)\right) \\
< & \xi\left(\Im\left(\wp_{m_{k}}\right), \Im\left(\wp_{m_{k}-1}\right)\right)+\xi\left(\Im\left(\wp_{m_{k}-1}\right), \Im\left(\wp_{m_{k}}\right)\right) \\
& +\epsilon+\xi\left(\Im\left(\wp_{n_{k}-1}\right), \Im\left(\wp_{n_{k}}\right)\right) .
\end{aligned}
$$

If $k \rightarrow+\infty$, and by (9), we can record

$$
\lim _{k \rightarrow+\infty} \xi\left(\Im\left(\wp_{m_{k}}\right), \Im\left(\wp_{n_{k}}\right)\right) \leq \lim _{k \rightarrow+\infty} \xi\left(\Im\left(\wp_{m_{k}-1}\right), \Im\left(\wp_{n_{k}-1}\right)\right) \leq \epsilon .
$$

Likewise, we have

$$
\lim _{k \rightarrow+\infty} \xi\left(\Im\left(\hbar_{m_{k}}\right), \Im\left(\hbar_{n_{k}}\right)\right) \leq \lim _{k \rightarrow+\infty} \xi\left(\Im\left(\hbar_{m_{k}-1}\right), \Im\left(\hbar_{n_{k}-1}\right)\right) \leq \epsilon
$$

and

$$
\lim _{k \rightarrow+\infty} \xi\left(\Im\left(\varpi_{m_{k}}\right), \Im\left(\varpi_{n_{k}}\right)\right) \leq \lim _{k \rightarrow+\infty} \xi\left(\Im\left(\varpi_{m_{k}-1}\right), \Im\left(\varpi_{n_{k}-1}\right)\right) \leq \epsilon
$$

Applying (10) and (12)-(14), we observe that

$$
\lim _{k \rightarrow+\infty} \max \left\{\begin{array}{l}
\xi\left(\Im\left(\wp_{m_{k}-1}\right), \Im\left(\wp_{n_{k}-1}\right)\right), \\
\xi\left(\Im\left(\hbar_{m_{k}-1}\right), \Im\left(\hbar_{n_{k}-1}\right)\right), \\
\xi\left(\Im\left(\varlimsup_{m_{k}-1}\right), \Im\left(\varlimsup_{n_{k}-1}\right)\right)
\end{array}\right\}=\epsilon
$$

Now, by stipulation (1), we can get

$$
\begin{aligned}
& \xi\left(\Im\left(\wp m_{k}\right), \Im\left(\wp_{n_{k}}\right)\right) \\
& =\xi\left(\Re\left(\wp_{m_{k}-1}, \hbar_{m_{k}-1}, \partial_{m_{k}-1}\right), \Re\left(\wp_{n_{k}-1}, \hbar_{n_{k}-1}, \check{\partial}_{n_{k}-1}\right)\right) \\
& \leq \Lambda\left(\begin{array}{c}
\pi\left(\max \left\{\xi\left(\Im\left(\wp_{m_{k}-1}\right), \Im\left(\wp_{n_{k}-1}\right)\right), \xi\left(\Im\left(\hbar_{m_{k}-1}\right), \Im\left(\hbar_{n_{k}-1}\right)\right), \xi\left(\Im\left(\varpi_{m_{k}-1}\right), \Im\left(\varpi_{n_{k}-1}\right)\right)\right\}\right), \\
\zeta \max \left\{\xi\left(\Im\left(\wp_{m_{k}-1}\right), \Im\left(\wp_{n_{k}-1}\right)\right), \xi\left(\Im\left(\hbar_{m_{k}-1}\right), \Im\left(\hbar_{n_{k}-1}\right)\right), \xi\left(\Im\left(\varpi_{m_{k}-1}\right), \Im\left(\varpi_{n_{k}-1}\right)\right)\right\}
\end{array}\right) \\
& \leq \pi\left(\operatorname { m a x } \left\{\xi\left(\Im\left(\wp_{m_{k}-1}\right), \Im\left(\wp_{n_{k}-1}\right)\right), \xi\left(\Im\left(\hbar_{m_{k}-1}\right),\right.\right.\right. \\
& \left.\left.\left.\Im\left(\hbar_{n_{k}-1}\right)\right), \xi\left(\Im\left(\varpi_{m_{k}-1}\right), \Im\left(\varpi_{n_{k}-1}\right)\right)\right\}\right), \\
& \xi\left(\Im\left(\hbar_{m_{k}}\right), \Im\left(\hbar_{n_{k}}\right)\right) \\
& =\xi\left(\Re\left(\hbar_{m_{k}-1}, \wp_{m_{k}-1}, \hbar_{m_{k}-1}\right), \Re\left(\hbar_{n_{k}-1}, \wp_{n_{k}-1}, \hbar_{n_{k}-1}\right)\right) \\
& \leq \Lambda\left(\begin{array}{c}
\pi\left(\max \left\{\xi\left(\Im\left(\hbar_{m_{k}-1}\right), \Im\left(\hbar_{n_{k}-1}\right)\right), \xi\left(\Im\left(\wp_{m_{k}-1}\right), \Im\left(\wp_{n_{k}-1}\right)\right)\right\}\right), \\
\zeta \max \left\{\xi\left(\Im\left(\hbar_{m_{k}-1}\right), \Im\left(\hbar_{n_{k}-1}\right)\right), \xi\left(\Im\left(\wp_{m_{k}-1}\right), \Im\left(\wp_{n_{k}-1}\right)\right)\right\}
\end{array}\right) \\
& \leq \pi\left(\max \left\{\xi\left(\Im\left(\hbar_{m_{k}-1}\right), \Im\left(\hbar_{n_{k}-1}\right)\right), \xi\left(\Im\left(\wp_{m_{k}-1}\right), \Im\left(\wp_{n_{k}-1}\right)\right)\right\}\right),
\end{aligned}
$$


and

$$
\begin{aligned}
& \xi\left(\Im\left(\varlimsup_{m_{k}}\right), \Im\left(\varlimsup_{n_{k}}\right)\right) \\
& =\xi\left(\Re\left(\varlimsup_{m_{k}-1}, \hbar_{m_{k}-1}, \wp_{m_{k}-1}\right), \Re\left(ð_{n_{k}-1}, \hbar_{n_{k}-1}, \wp_{n_{k}-1}\right)\right) \\
& \leq \Lambda\left(\begin{array}{c}
\pi\left(\max \left\{\xi\left(\Im\left(\varpi_{m_{k}-1}\right), \Im\left(\varpi_{n_{k}-1}\right)\right), \xi\left(\Im\left(\hbar_{m_{k}-1}\right), \Im\left(\hbar_{n_{k}-1}\right)\right), \xi\left(\Im\left(\wp_{m_{k}-1}\right), \Im\left(\wp_{n_{k}-1}\right)\right)\right\}\right), \\
\zeta \max \left\{\xi\left(\Im\left(\varpi_{m_{k}-1}\right), \Im\left(\varpi_{n_{k}-1}\right)\right), \xi\left(\Im\left(\hbar_{m_{k}-1}\right), \Im\left(\hbar_{n_{k}-1}\right)\right), \xi\left(\Im\left(\wp_{m_{k}-1}\right), \Im\left(\wp_{n_{k}-1}\right)\right)\right\}
\end{array}\right) \\
& \leq \pi\left(\operatorname { m a x } \left\{\xi\left(\Im\left(\varpi_{m_{k}-1}\right), \Im\left(\varpi_{n_{k}-1}\right)\right), \xi\left(\Im\left(\hbar_{m_{k}-1}\right), \Im\left(\hbar_{n_{k}-1}\right)\right),\right.\right. \\
& \left.\left.\xi\left(\Im\left(\wp_{m_{k}-1}\right), \Im\left(\wp_{n_{k}-1}\right)\right)\right\}\right) \text {. }
\end{aligned}
$$

The three inequalities (16)-(18) say that

$$
\begin{array}{r}
\max \left\{\xi\left(\Im\left(\wp_{m_{k}}\right), \Im\left(\wp_{n_{k}}\right)\right), \xi\left(\Im\left(\hbar_{m_{k}}\right), \Im\left(\hbar_{n_{k}}\right)\right), \xi\left(\Im\left(\varpi_{m_{k}}\right), \Im\left(\varpi_{n_{k}}\right)\right)\right\} \\
\quad \leq \pi\left(\max \left\{\begin{array}{c}
\xi\left(\Im\left(\Im_{m_{k}-1}\right), \Im\left(\varlimsup_{n_{k}-1}\right)\right), \xi\left(\Im\left(\hbar_{m_{k}-1}\right), \Im\left(\hbar_{n_{k}-1}\right)\right), \\
\xi\left(\Im\left(\wp_{m_{k}-1}\right), \Im\left(\wp_{n_{k}-1}\right)\right)
\end{array}\right\}\right) .
\end{array}
$$

Letting $k \rightarrow+\infty$ in (19) and having in mind (15), we get

$$
0<\epsilon \leq \lim _{k \rightarrow+\infty} \max \left\{\begin{array}{l}
\xi\left(\Im\left(\wp_{m_{k}-1}\right), \Im\left(\wp_{n_{k}-1}\right)\right), \\
\xi\left(\Im\left(\hbar_{m_{k}-1}\right), \Im\left(\hbar_{n_{k}-1}\right)\right), \\
\xi\left(\Im\left(\Im_{m_{k}-1}\right), \Im\left(\varlimsup_{n_{k}-1}\right)\right)
\end{array}\right\} \leq \lim _{\nu \rightarrow \epsilon^{+}} \pi(\nu)<\epsilon .
$$

Incompatibility. Hence $\left\{\Im\left(\wp_{n}\right)\right\},\left\{\Im\left(\hbar_{n}\right)\right\}$, and $\left\{\Im\left(\varpi_{n}\right)\right\}$ are Cauchy sequences in a POCML space. By completeness, there are $\wp, \hbar, \partial \in \chi$ such that

$$
\lim _{n \rightarrow+\infty} \Im\left(\wp_{n}\right)=\wp, \quad \lim _{n \rightarrow+\infty} \Im\left(\hbar_{n}\right)=\hbar, \quad \text { and } \quad \lim _{n \rightarrow+\infty} \Im\left(\varpi_{n}\right)=ð
$$

Applying the thought of continuity of $\Im$ on (20), we can get

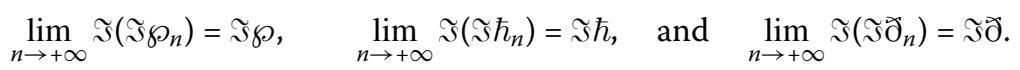

Since $\Im$ commutes with $\Re$, then by (3) we can write

$$
\left\{\begin{array}{l}
\Im\left(\Im \wp_{n+1}\right)=\Im\left(\Re\left(\wp_{n}, \hbar_{n}, \coprod_{n}\right)\right)=\Re\left(\Im \wp_{n}, \Im \hbar_{n}, \Im \partial_{n}\right), \\
\Im\left(\Im \hbar_{n+1}\right)=\Im\left(\Re\left(\hbar_{n}, \wp_{n}, \hbar_{n}\right)\right)=\Re\left(\Im \hbar_{n}, \Im \wp_{n}, \Im \hbar_{n}\right), \\
\Im\left(\Im \partial_{n+1}\right)=\Im\left(\Re\left(\coprod_{n}, \hbar_{n}, \wp_{n}\right)\right)=\Re\left(\Im \partial_{n}, \Im \hbar_{n}, \Im \wp_{n}\right) .
\end{array}\right.
$$

Letting $n \rightarrow+\infty$ in (22) and taking into account relations (20)-(21) and the continuity of $\mathfrak{R}$, we have

$$
\begin{aligned}
& \Im \wp=\lim _{n \rightarrow+\infty} \Im\left(\Im \wp_{n+1}\right)=\lim _{n \rightarrow+\infty} \Im\left(\Re\left(\wp_{n}, \hbar_{n}, \widetilde{\partial}_{n}\right)\right) \\
& =\Re\left(\lim _{n \rightarrow+\infty} \Im \wp_{n}, \lim _{n \rightarrow+\infty} \Im \hbar_{n}, \lim _{n \rightarrow+\infty} \Im \Im_{n}\right)=\Re(\wp, \hbar, ð), \\
& \Im \hbar=\lim _{n \rightarrow+\infty} \Im\left(\Im \hbar_{n+1}\right)=\lim _{n \rightarrow+\infty} \Im\left(\Re\left(\hbar_{n}, \wp_{n}, \hbar_{n}\right)\right)
\end{aligned}
$$




$$
\begin{aligned}
& =\Re\left(\lim _{n \rightarrow+\infty} \Im \hbar_{n}, \lim _{n \rightarrow+\infty} \Im \wp_{n}, \lim _{n \rightarrow+\infty} \Im \hbar_{n}\right)=\Re(\hbar, \wp, \hbar), \\
\Im \Im & =\lim _{n \rightarrow+\infty} \Im\left(\Im \Im_{n+1}\right)=\lim _{n \rightarrow+\infty} \Im\left(\Re\left(\Im_{n}, \hbar_{n}, \wp_{n}\right)\right) \\
& =\Re\left(\lim _{n \rightarrow+\infty} \Im \coprod_{n}, \lim _{n \rightarrow+\infty} \Im \hbar_{n}, \lim _{n \rightarrow+\infty} \Im \wp_{n}\right)=\Re(\Im, \hbar, \wp) .
\end{aligned}
$$

Thus, there is a tripled coincidence point of $\Re$ and $\mathfrak{I}$. This ends the demonstration.

The question arises here. What happens when you omit the continuity stipulation of the mapping $\Re$ ? To answer this query, we give the following theorem.

Theorem 3.2 Let $(\chi, \precsim, \xi)$ be a POCML space. Assume that $\Re: \chi^{3} \rightarrow \chi$ and $\Im: \chi \rightarrow \chi$ fulfill the following hypotheses:

(i) $\Re\left(\chi^{3}\right) \subseteq \Im(\chi)$;

(ii) $\Re$ has a mixed $\Im$-monotone property;

(iii) $(\Im(\chi), \xi)$ is a complete metric-like space and $\chi$ is obligated by the following assumptions:

(I) $l_{n} \precsim l$ if a nondecreasing sequence $l_{n} \rightarrow l, n \rightarrow+\infty$,

(II) $j_{n} \succsim j$ if a nonincreasing sequence $j_{n} \rightarrow j, n \rightarrow+\infty$;

(iv) $\Im$ is continuous and commutes with $\Re$;

(v) There are $\pi \in \Pi, \zeta \geq 0$, and $\Lambda \in \complement$ such that

$$
\begin{aligned}
& \xi(\Re(\wp, \hbar, ð), \Re(x, y, z)) \\
& \quad \leq \Lambda\left(\begin{array}{c}
\pi(\max \{\xi(\Im(\wp), \Im(x)), \xi(\Im(\hbar), \Im(y)), \xi(\Im(ð), \Im(z))\}), \\
\zeta \max \{\xi(\Im(\wp), \Im(x)), \xi(\Im(\hbar), \Im(y)), \xi(\Im(\Im), \Im(z))\}
\end{array}\right) .
\end{aligned}
$$

If there exist $\wp_{\circ}, \hbar_{\circ}, \partial_{\circ} \in \chi$ such that $\Im\left(\wp_{\circ}\right) \precsim \Re\left(\wp_{\circ}, \hbar_{\circ}, \partial_{\circ}\right), \Im\left(\hbar_{\circ}\right) \succsim \Re\left(\hbar_{\circ}, \wp_{\circ}, \check{\partial}_{\circ}\right)$, and $\Im\left(\varpi_{\circ}\right) \precsim \Re\left(\varpi_{\circ}, \hbar_{\circ}, \wp_{\circ}\right)$, then $\Re$ and $\Im$ have a tripled coincidence point.

Proof The same scenario of Theorem 3.1 implies that $\left\{\Im\left(\wp_{n}\right)\right\},\left\{\Im\left(\hbar_{n}\right)\right\}$, and $\left\{\Im\left(\Im_{n}\right)\right\}$ are Cauchy sequences in a complete metric-like space $(\Im(\chi), \xi)$. Then there are $\wp, \hbar, \partial \in \chi$ such that (20) is achieved. By nondecreasing of $\left\{\Im\left(\wp_{n}\right)\right\}$ and $\left\{\Im\left(\Im_{n}\right)\right\}$, nonincreasing of $\left\{\Im\left(\hbar_{n}\right)\right\}$, and properties (I) and (II) of $\chi$, we can write

$$
\Im\left(\wp_{n}\right) \precsim \Im(\wp), \quad \Im\left(\hbar_{n}\right) \succsim \Im(\hbar), \quad \text { and } \quad \Im\left(\Im_{n}\right) \precsim \Im(ð) \quad \text { for all } n \geq 0 \text {. }
$$

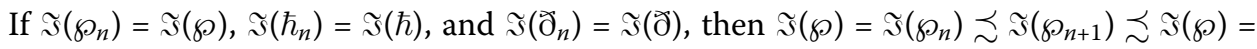
$\Im\left(\wp_{n}\right), \Im(\hbar) \precsim \Im\left(\hbar_{n+1}\right) \precsim \Im\left(\hbar_{n}\right)=\Im(\hbar)$, and $\Im(ð)=\Im\left(\varpi_{n}\right) \precsim \Im\left(\partial_{n+1}\right) \precsim \Im(ð)=\Im\left(\partial_{n}\right)$. This leads to

$$
\Im\left(\wp_{n}\right)=\Im\left(\wp_{n+1}\right)=\Re\left(\wp_{n}, \hbar_{n}, \breve{\partial}_{n}\right), \quad \Im\left(\hbar_{n}\right)=\Im\left(\hbar_{n+1}\right)=\Re\left(\hbar_{n}, \check{\partial}_{n}, \wp_{n}\right),
$$

and

$$
\Im\left(\coprod_{n}\right)=\Im\left(\coprod_{n+1}\right)=\Re\left(\varlimsup_{n}, \wp_{n}, \hbar_{n}\right) .
$$


Accordingly, the trio $\left(\wp_{n}, \hbar_{n}, \partial_{n}\right)$ is a tripled coincidence point of $\Im$ and $\Re$. So, we postulate, for all $n \geq 0,\left(\Im \wp_{n}, \Im \hbar_{n}, \Im \coprod_{n}\right) \neq(\Im \wp, \Im \hbar, \Im ð)$; consequently, by (1) we can get

$$
\begin{aligned}
\xi & (\Im \wp, \Re(\wp, \hbar, \Im)) \\
& \leq \xi\left(\Im \wp, \Im \wp_{n+1}\right)+\xi\left(\Im \wp_{n+1}, \Re(\wp, \hbar, \Im)\right) \\
& =\xi\left(\Im \wp, \Im \wp_{n+1}\right)+\xi\left(\Re\left(\wp_{n}, \hbar_{n}, \partial_{n}\right), \Re(\wp, \hbar, \Im)\right) \\
& \leq \xi\left(\Im \wp, \Im \wp_{n+1}\right)+\Lambda\left(\begin{array}{c}
\pi\left(\max \left\{\xi(\Im(\wp), \Im(\wp)), \xi\left(\Im\left(\hbar_{n}\right), \Im(\hbar)\right), \xi\left(\Im\left(\Im_{n}\right), \Im(\Im)\right)\right\}\right), \\
\zeta \max \left\{\xi\left(\Im\left(\wp_{n}\right), \Im(\wp)\right), \xi\left(\Im\left(\hbar_{n}\right), \Im(\hbar)\right), \xi\left(\Im\left(\Im_{n}\right), \Im(ð)\right)\right\}
\end{array}\right) \\
& \leq \xi\left(\Im \wp, \Im \wp_{n+1}\right)+\pi\left(\max \left\{\xi\left(\Im\left(\wp_{n}\right), \Im(\wp)\right), \xi\left(\Im\left(\hbar_{n}\right), \Im(\hbar)\right), \xi\left(\Im\left(\Im_{n}\right), \Im(\Im)\right)\right\}\right) .
\end{aligned}
$$

Letting $n \rightarrow+\infty$ in (23) and using (20), we deduce that $\xi(\Im \wp, \Re(\wp, \hbar, ð))=0$, thus $\Im \wp=$

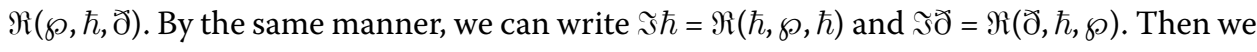
have reached the end of the proof.

Mathematicians in this direction can stir up the following: What about the structure and exclusivity of a tripled combined fixed point? To reduce this excitement, we recognize a partial ordering $(\chi, \leq)$ as follows: For all $(\wp, \hbar, ð)$ and $(x, y, z)$ belonging to the product $\chi^{3}$,

$$
(\wp, \hbar, ð) \leq(x, y, z) \quad \text { if and only if } \wp \leq x, \quad \hbar \geq y, \quad \partial \leq z .
$$

Let us say that the trios $(\wp, \hbar, ð)$ and $(x, y, z)$ are comparable if

$$
(\wp, \hbar, ð) \leq(x, y, z) \quad \text { or } \quad(x, y, z) \leq(\wp, \hbar, ð) \text {; }
$$

also, $(\wp, \hbar, ð)$ is equal to $(x, y, z)$ iff $\wp=x, \hbar=y$, and $\varnothing=z$.

Now, the excitement is killed by the following important theorem.

Theorem 3.3 Besides the presumptions of Theorem 3.1, postulate for all $(\wp, \hbar, ð),(x, y, z) \in$ $\chi^{3}$ there exists $(\alpha, \beta, \gamma) \in \chi^{3}$ such that $(\Re(\alpha, \beta, \gamma), \Re(\beta, \alpha, \beta), \Re(\gamma, \beta, \alpha))$ is comparable to $(\Re(\wp, \hbar, \widetilde{\partial}), \Re(\hbar, \wp, \hbar), \Re(ð, \hbar, \wp))$ and $(\Re(x, y, z), \Re(y, x, y), \Re(z, y, x))$. Therefore, there is a unique tripled combined fixed point $(\wp, \hbar, \precsim)$ for the mappings $\Re$ and $\Im$, i.e.,

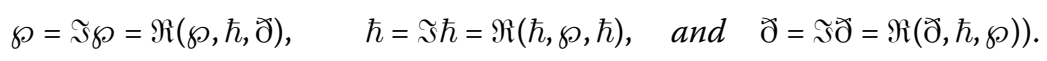

Proof According to Theorem 3.1, the set of tripled coincidence points of $\Re$ and $\Im$ is nonempty. Thence, we assume that $(\wp, \hbar, \precsim)$ and $(x, y, z)$ are two tripled coincidence points of $\Re$ and $\mathfrak{I}$, i.e.,

$$
\begin{array}{ll}
\Re(\wp, \hbar, ð)=\Im \wp, & \Re(x, y, z)=\Im x, \\
\Re(\hbar, \wp, \hbar)=\Im \hbar, & \Re(y, x, y)=\Im y, \\
\Re(ð, \hbar, \wp)=\Im ð, & \Re(z, y, x)=\Im z .
\end{array}
$$

First, we shall show that ( $\Im \wp, \Im \hbar$, $\Im ð)$ equals ( $\Im x, \Im y, \Im z)$. Consider the hypothesis of com-

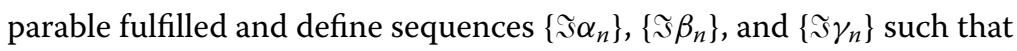

$$
\alpha_{n}=\alpha_{\circ}, \quad \beta_{n}=\beta_{\circ}, \quad \gamma_{n}=\gamma_{\circ},
$$


and for all $n \geq 1$,

$$
\begin{aligned}
& \Im \alpha_{n}=\Re\left(\alpha_{n-1}, \beta_{n-1}, \gamma_{n-1}\right), \\
& \Im \beta_{n}=\Re\left(\beta_{n-1}, \alpha_{n-1}, \beta_{n-1}\right), \\
& \Im \gamma_{n}=\Re\left(\gamma_{n-1}, \beta_{n-1}, \alpha_{n-1}\right) .
\end{aligned}
$$

On the other hand, appoint $\wp_{\circ}=\wp, \hbar_{\circ}=\hbar, \partial=\partial_{\circ}, x_{\circ}=x, y_{\circ}=y$, and $z=z_{\circ}$, and by the

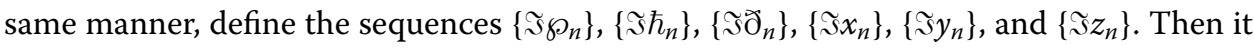
is easy to conclude that

$$
\begin{array}{ll}
\Im \wp_{n}=\Re(\wp, \hbar, ð), & \Im x_{n}=\Re(x, y, z), \\
\Im \hbar_{n}=\Re(\hbar, \wp, \hbar), & \Im y_{n}=\Re(y, x, y), \\
\Im \mho_{n}=\Re(ð, b, \wp), & \Im z_{n}=\Re(z, y, x),
\end{array}
$$

for all $n \geq 1$. Since $(\Re(\wp, \hbar, ð), \Re(\hbar, \wp, \hbar), \Re(ð, \hbar, \wp))=\left(\Im \wp_{1}, \Im \hbar_{1}, \Im \partial_{1}\right)=(\Im \wp, \Im \hbar, \Im ð)$

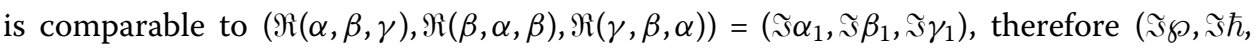
$\Im ð) \geq\left(\Im \alpha_{1}, \Im \beta_{1}, \Im \coprod_{1}\right)$, by repeating for all $n$, we get

$$
(\mathfrak{\wp}, \mathfrak{s} \hbar, \mathfrak{s} \partial) \succsim\left(\mathfrak{I} \alpha_{n}, \mathfrak{s} \beta_{n}, \mathfrak{s} \gamma_{n}\right)
$$

Applying (1), (25), and (24), we can write

$$
\begin{aligned}
\xi\left(\Im \alpha_{n+1}, \Im \wp\right) & =\xi\left(\Re\left(\alpha_{n}, \beta_{n}, \gamma_{n}\right), \Re(\wp, \hbar, ð)\right) \\
& \leq \Lambda\left(\begin{array}{c}
\pi\left(\max \left\{\xi\left(\Im\left(\left(\alpha_{n}\right), \Im(\wp)\right), \xi\left(\Im\left(\beta_{n}\right), \Im(\hbar)\right), \xi\left(\Im\left(\gamma_{n}\right), \Im(ð)\right)\right\}\right),\right. \\
\zeta \max \left\{\xi\left(\Im\left(\left(\alpha_{n}\right), \Im(\wp)\right), \xi\left(\Im\left(\beta_{n}\right), \Im(\hbar)\right), \xi\left(\Im\left(\gamma_{n}\right), \Im(ð)\right)\right\}\right.
\end{array}\right) \\
& \leq \pi\left(\max \left\{\xi\left(\Im\left(\left(\alpha_{n}\right), \Im(\wp)\right), \xi\left(\Im\left(\beta_{n}\right), \Im(\hbar)\right), \xi\left(\Im\left(\gamma_{n}\right), \Im(ð)\right)\right\}\right),\right. \\
\xi\left(\Im \hbar, \Im \beta_{n+1}\right) & =\xi\left(\Re(\hbar, \wp, \hbar), \Re\left(\beta_{n}, \alpha_{n}, \beta_{n}\right)\right) \\
& \leq \Lambda\left(\begin{array}{c}
\pi\left(\max \left\{\xi\left(\Im(\hbar), \Im\left(\beta_{n}\right)\right), \xi\left(\Im(\wp), \Im\left(\alpha_{n}\right)\right)\right\}\right), \\
\zeta \max \left\{\xi\left(\Im(\hbar), \Im\left(\beta_{n}\right)\right), \xi\left(\Im(\wp), \Im\left(\alpha_{n}\right)\right)\right\}
\end{array}\right) \\
& \leq \pi\left(\max \left\{\xi\left(\Im(\hbar), \Im\left(\beta_{n}\right)\right), \xi\left(\Im(\wp), \Im\left(\alpha_{n}\right)\right)\right\}\right) \\
& \leq \pi\left(\max \left\{\begin{array}{c}
\xi\left(\Im(\hbar), \Im\left(\beta_{n}\right)\right), \xi\left(\Im(\wp), \Im\left(\alpha_{n}\right)\right), \\
\xi\left(\Theta(ð), \Theta\left(\gamma_{n}\right)\right)
\end{array}\right\}\right),
\end{aligned}
$$

and

$$
\begin{aligned}
\xi\left(\Im ð, \Im \gamma_{n+1}\right) & =\xi\left(\Re(\Im, \hbar, \wp), \Re\left(\gamma_{n}, \beta_{n}, \alpha_{n}\right)\right) \\
& \leq \Lambda\left(\begin{array}{c}
\pi\left(\max \left\{\xi\left(\Im(ð), \Im\left(\gamma_{n}\right)\right), \xi\left(\Im(\hbar), \Im\left(\beta_{n}\right)\right), \xi\left(\Im(\wp), \Im\left(\alpha_{n}\right)\right)\right\}\right), \\
\zeta \max \left\{\xi\left(\Im(\Im), \Im\left(\gamma_{n}\right)\right), \xi\left(\Im(\hbar), \Im\left(\beta_{n}\right)\right), \xi\left(\Im(\wp), \Im\left(\alpha_{n}\right)\right)\right\}
\end{array}\right) \\
& \leq \pi\left(\max \left\{\xi\left(\Im(\Im), \Im\left(\gamma_{n}\right)\right), \xi\left(\Im(\hbar), \Im\left(\beta_{n}\right)\right), \xi\left(\Im(\wp), \Im\left(\alpha_{n}\right)\right)\right\}\right) .
\end{aligned}
$$


Inequalities (26)-(28) indicate that

$$
\begin{aligned}
& \max \left\{\xi\left(\Im \alpha_{n+1}, \Im \wp\right), \xi\left(\Im \hbar, \Im \beta_{n+1}\right), \xi\left(\Im \partial, \Im \gamma_{n+1}\right)\right\} \\
& \quad \leq \pi\left(\max \left\{\xi\left(\Im(\Im), \Im\left(\gamma_{n}\right)\right), \xi\left(\Im(\hbar), \Im\left(\beta_{n}\right)\right), \xi\left(\Im(\wp), \Im\left(\alpha_{n}\right)\right)\right\}\right) .
\end{aligned}
$$

Subsequently, for all $n \geq 1$, we can get

$$
\begin{aligned}
& \max \left\{\xi\left(\Im(\Im), \Im\left(\gamma_{n}\right)\right), \xi\left(\Im(\hbar), \Im\left(\beta_{n}\right)\right), \xi\left(\Im(\wp), \Im\left(\alpha_{n}\right)\right)\right\} \\
& \quad \leq \pi^{n}\left(\max \left\{\xi\left(\Im(\Im), \Im\left(\gamma_{\circ}\right)\right), \xi\left(\Im(\hbar), \Im\left(\beta_{\circ}\right)\right), \xi\left(\Im(\wp), \Im\left(\alpha_{\circ}\right)\right)\right\}\right) .
\end{aligned}
$$

Since $\pi(\iota)<\iota$ and $\lim _{\rho \rightarrow \iota^{+}} \pi(\rho)<\iota$, then for all $\iota>0, \lim _{n \rightarrow+\infty} \pi^{n}(\iota)=0$. Thus, assign this on (29), after letting $n \rightarrow+\infty$, we have

$$
\lim _{n \rightarrow+\infty} \max \left\{\xi\left(\Im(\Im), \Im\left(\gamma_{n}\right)\right), \xi\left(\Im(\hbar), \Im\left(\beta_{n}\right)\right), \xi\left(\Im(\wp), \Im\left(\alpha_{n}\right)\right)\right\}=0
$$

yields

$$
\lim _{n \rightarrow+\infty} \xi\left(\Im(\Im), \Im\left(\gamma_{n}\right)\right)=\lim _{n \rightarrow+\infty} \xi\left(\Im(\hbar), \Im\left(\beta_{n}\right)\right)=\lim _{n \rightarrow+\infty} \xi\left(\Im(\wp), \Im\left(\alpha_{n}\right)\right)=0 .
$$

In a similar way, we can write

$$
\lim _{n \rightarrow+\infty} \xi\left(\Im z, \Im \gamma_{n}\right)=\lim _{n \rightarrow+\infty} \xi\left(\Im y, \Im \beta_{n}\right)=\lim _{n \rightarrow+\infty} \xi\left(\Im \alpha_{n}, \Im x\right)=0
$$

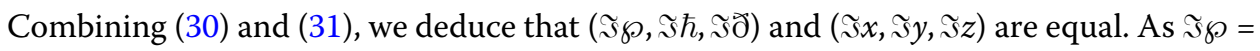

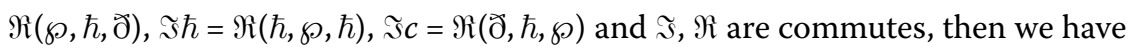

$$
\begin{aligned}
& \Im \wp^{*}=\Im(\Im \wp)=\Im(\Re(\wp, \hbar, ठ))=\Re(\Im \wp, \Im \hbar, \Im ð), \\
& \Im \hbar^{*}=\Im(\Im \hbar)=\Im(\Re(\hbar, \wp, \hbar))=\Re(\Im \hbar, \Im \wp, \Im \hbar), \\
& \Im \nearrow^{*}=\Im(\Im ð)=\Im(\Re(ð, \hbar, \wp))=\Re(\Im \partial, \Im \hbar, \Im \wp),
\end{aligned}
$$

where $a^{*}=\Im \wp, \hbar^{*}=\Im \hbar$, and $\Im^{*}=\Im ð$. Therefore the trio $\left(\wp^{*}, \hbar^{*}, \supset^{*}\right)$ is a tripled coincidence point of $\Im$ and $\Re$. Hence, $\left(\Im \wp^{*}, \Im \hbar^{*}, \Im \Im^{*}\right)$ and $(\Im \wp, \Im \hbar, \Im \Im)$ are equal, so, one can write

$$
\Im \wp^{*}=\Im \wp=\wp^{*}, \quad \Im \hbar^{*}=\Im \hbar=\hbar^{*}, \quad \text { and } \quad \Im \nearrow^{*}=\Im ð=ð^{*} .
$$

Thus, $\left(\wp^{*}, \hbar^{*}, \partial^{*}\right)$ is a tripled common fixed of $\Im$ and $\Re$. The uniqueness follows immediately by (1).

It is known that the numerical examples clarify and strengthen the theoretical results, so we shall present some examples in what follows.

Example 3.4 Let $\Lambda(\lambda, \mu)=\varrho \lambda, 0<\varrho<1$, and $\chi=\mathbb{R}$ be equipped with

$$
\xi(\wp, \hbar)=\wp+\hbar
$$


for all $\wp, \hbar \in \chi$. Define the order relation $\leq$ by

$$
\left(\wp \leq_{\chi} \hbar \Longleftrightarrow \wp=\hbar\right) \quad \text { or } \quad(\wp, \hbar \in[0,1] \text { and } \wp \leq \hbar)
$$

It is clear that $(\Im(\chi), \xi)$ is a complete metric-like space. Define $\Im: \chi \rightarrow \chi$ and $\Re: \chi^{3} \rightarrow \chi$ by

$$
\begin{aligned}
& \Im(\wp)= \begin{cases}\frac{3}{2} \wp & \text { if } \wp<0, \\
\wp & \text { if } \wp \in[0,1], \\
\frac{4}{3} \wp & \text { if } \wp>1,\end{cases} \\
& \Re(\wp, \hbar, ð)=\frac{\wp+\hbar+\partial}{8} .
\end{aligned}
$$

It is obvious that $\Re\left(\chi^{3}\right) \subset \Im(\chi), \Re$ has a mixed $\Im$-monotone property. Now we will go to investigate the contractive condition of Theorem 3.2 for all $\wp, \hbar, ð, x, y, z \in \chi$ such that $\Im \wp \leq_{\chi} \Im x$, $\Im y \leq_{\chi} \Im \hbar$, and $\Im ð \leq_{\Upsilon} \Im z$. Take $\pi(v)=\frac{1}{2} v$ for all $v \in[0,+\infty), \zeta \geq 0$, and $\varrho=\frac{3}{4}$. Now, we check the following statuses:

Status $i$. If $\wp, \hbar, \precsim, x, y, z \in[0,1]$, we have

$$
\begin{aligned}
\xi(\Re(\wp, \hbar, ð), \Re(x, y, z)) & =\frac{\wp+\hbar+\partial}{8}+\frac{x+y+z}{8} \\
& =\frac{\wp+x}{8}+\frac{\hbar+y}{8}+\frac{c+z}{8} \\
& \leq \frac{3}{8} \max \{\wp+x, \hbar+x, ð+z\} \\
& =\frac{3}{4} \times \frac{1}{2} \max \{\wp+x, \hbar+y, ð+z\} \\
& =\frac{3}{4} \pi(\max \{\xi(\Im(\wp), \Im(x)), \xi(\Im(\hbar), \Im(y)), \xi(\Im(\Im), \Im(z))\}) \\
& =\Lambda\left(\begin{array}{c}
\pi(\max \{\xi(\Im(\wp), \Im(x)), \xi(\Im(\hbar), \Im(y)), \xi(\Im(\Im), \Im(z))\}), \\
\zeta \max \{\xi(\Im(\wp), \Im(x)), \xi(\Im(\hbar), \Im(y)), \xi(\Im(ð), \Im(z))\}
\end{array}\right) .
\end{aligned}
$$

- Status ii. If $\wp, x, \hbar, y \in[0,1], \precsim, z \notin[0,1]$, here $\Im ð, \Im z \notin[0,1]$ and since they must be

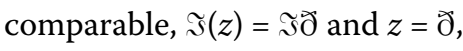

$$
\begin{aligned}
\xi(\Re(\wp, \hbar, ð), \Re(x, y, z)) & =\frac{\wp+\hbar+ð}{8}+\frac{x+y+z}{8} \\
& =\frac{\wp+x}{8}+\frac{\hbar+y}{8}+\frac{2 z}{8} \\
& \leq \frac{3}{8} \max \{\wp+x, \hbar+x, 2 z\} \\
& \leq \frac{3}{8}\left\{\begin{array}{l}
\max \{\wp+x, \hbar+x, 3 z\} \quad \text { if } \wp<0, \\
\max \left\{\wp+x, \hbar+x, \frac{8}{3} z\right\} \quad \text { if } \wp>1
\end{array}\right. \\
& =\frac{3}{4} \times \frac{1}{2} \max \{\xi(\Im(\wp), \Im(x)), \xi(\Im(\hbar), \Im(y)), \xi(\Im(\Im), \Im(z))\}
\end{aligned}
$$




$$
\begin{aligned}
& =\frac{3}{4} \pi(\max \{\xi(\Im(\wp), \Im(x)), \xi(\Im(\hbar), \Im(y)), \xi(\Im(ð), \Im(z))\}) \\
& =\Lambda\left(\begin{array}{c}
\pi(\max \{\xi(\Im(\wp), \Im(x)), \xi(\Im(\hbar), \Im(y)), \xi(\Im(\Im), \Im(z))\}), \\
\zeta \max \{\xi(\Im(\wp), \Im(x)), \xi(\Im(\hbar), \Im(y)), \xi(\Im(ð), \Im(z))\}
\end{array}\right) .
\end{aligned}
$$

Status iii. If $(\wp, x, ð, z \in[0,1]$ and $\hbar, y \notin[0,1])$, or $(\hbar, y, ð, z \in[0,1]$ and $\wp, x \notin[0,1])$, we have the same results of Status ii.

- Status iv. If $\wp, x \in[0,1]$ and $\hbar, y, \partial, z \notin[0,1]$, here $\Im \hbar, \Im y, \Im ð, \Im z \notin[0,1]$ and since they

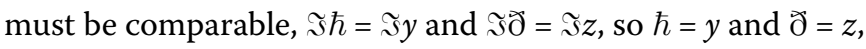

$$
\begin{aligned}
\xi(\Re(\wp, \hbar, ð), \Re(x, y, z)) & =\frac{\wp+\hbar+\partial}{8}+\frac{x+y+z}{8} \\
& =\frac{\wp+x}{8}+\frac{2 y}{8}+\frac{2 z}{8} \\
& \leq \frac{3}{8} \max \{a+x, 2 y, 2 z\} \\
& \leq \frac{3}{8}\left\{\begin{array}{l}
\max \{\wp+x, 3 y, 3 z\} \quad \text { if } a<0, \\
\max \left\{\wp+x, \frac{8}{3} y, \frac{8}{3} z\right\} \quad \text { if } a>1
\end{array}\right. \\
& =\frac{3}{4} \times \frac{1}{2} \max \{\xi(\Im(\wp), \Im(x)), \xi(\Im(b), \Im(y)), \xi(\Im(\Im), \Im(z))\} \\
& =\frac{3}{4} \pi(\max \{\xi(\Im(\wp), \Im(x)), \xi(\Im(b), \Im(y)), \xi(\Im(\Im), \Im(z))\}) \\
& =\Lambda\left(\begin{array}{c}
\pi(\max \{\xi(\Im(\wp), \Im(x)), \xi(\Im(b), \Im(y)), \xi(\Im(\Im), \Im(z))\}), \\
\zeta \max \{\xi(\Im(\wp), \Im(x)), \xi(\Im(b), \Im(y)), \xi(\Im(\Im), \Im(z))\}
\end{array}\right) .
\end{aligned}
$$

- Status $v$. If $(\hbar, y \in[0,1]$ and $\wp, x, \partial, z \notin[0,1])$ or $(ð, z \in[0,1]$ and $\wp, x, \hbar, y \notin[0,1])$, we treat it analogously to Status iv.

- Status vi. If $\wp, x, \hbar, y, \partial, z \notin[0,1]$, then the only possibility for $\Im x, \Im \wp$ as well as $\Im y, \Im \hbar$ and $\Im z$, ऽð to be comparable is that $x=\wp, y=\hbar$, and $z=ð$,

$$
\begin{aligned}
\xi(\Re(\wp, \hbar, ð), \Re(x, y, z)) & =\frac{\wp+\hbar+ð}{8}+\frac{x+y+z}{8} \\
& =\frac{2 x}{8}+\frac{2 y}{8}+\frac{2 z}{8} \\
& \leq \frac{3}{8} \max \{2 x, 2 y, 2 z\} \\
& \leq \frac{3}{8}\left\{\begin{array}{l}
\max \{3 x, 3 y, 3 z\} \quad \text { if } a<0, \\
\max \left\{\frac{8}{3} x, \frac{8}{3} y, \frac{8}{3} z\right\} \quad \text { if } a>1
\end{array}\right. \\
& =\frac{3}{4} \times \frac{1}{2} \max \{\xi(\Im(\wp), \Im(x)), \xi(\Im(\hbar), \Im(y)), \xi(\Im(\Im), \Im(z))\} \\
& =\frac{3}{4} \pi(\max \{\xi(\Im(\wp), \Im(x)), \xi(\Im(\hbar), \Im(y)), \xi(\Im(\Im), \Im(z))\}) \\
& =\Lambda\left(\begin{array}{l}
\pi(\max \{\xi(\Im(\wp), \Im(x)), \xi(\Im(\hbar), \Im(y)), \xi(\Im(\Im), \Im(z))\}), \\
\zeta \max \{\xi(\Im(\wp), \Im(x)), \xi(\Im(\hbar), \Im(y)), \xi(\Im(\Im), \Im(z))\}
\end{array}\right) .
\end{aligned}
$$


The six statuses complete postulates of Theorem 3.2 and $(0,0,0)$ is a tripled coincidence common fixed point of $\Im$ and $\Re$.

Example 3.5 Let $\Lambda(\lambda, \mu)=\lambda-\mu$ and $\chi=[0,+\infty)$ be equipped with

$$
\xi(\wp, \hbar)=\max \{\wp, \hbar\}
$$

for all $\wp, \hbar \in \chi$. Define the order relation by

$$
\wp, \hbar \in \Upsilon, \quad\left(\wp \leq_{\chi} \hbar \Longleftrightarrow \wp=\hbar=0\right) \quad \text { or } \quad(\wp, \hbar \in(0,+\infty) \text { and } \wp \leq \hbar)
$$

It is obvious that $(\Im(\chi), \xi)$ is a complete metric-like space. Let us define $\Im: \chi \rightarrow \chi$ and $\Re: \chi^{3} \rightarrow \chi$ by

$$
\chi(\wp)=\frac{1}{128} \wp, \quad \text { and } \quad \Re(\wp, \hbar, \partial)= \begin{cases}\frac{1}{130} & \text { if } \wp \hbar ð \neq 0, \\ 0 & \text { if } \wp \hbar \partial=0\end{cases}
$$

for all $\wp, \hbar, \partial \in \chi$. It is evident that $\Re\left(\chi^{3}\right) \subset \Im(\chi), \Xi$ has a mixed $\Theta$-monotone property. Now, for all $\wp, \hbar, \partial, x, y, z \in \chi$, if $\wp \hbar \partial=0$ or $x y z=0$, then the contractive condition of Theorem 3.2 is verified directly, so we discuss it when $\wp \hbar \partial \neq 0$ or $x y z \neq 0$. Take into account $\pi(\nu)=\frac{1}{8} v$ for all $v \in[0,+\infty)$ and $\zeta=\frac{15}{128}$. Thus, we have

$$
\begin{aligned}
\xi(\Re(\wp, \hbar, ð), \Re(x, y, z))= & \frac{1}{130} \\
\leq & \frac{1}{128} \max \{\max \{\wp, x\}, \max \{\hbar, y\}, \max \{ð, z\}\} \\
= & \left(\frac{1}{8}-\frac{15}{128}\right) \max \{\xi(\Im(\wp), \Im(x)), \xi(\Im(\hbar), \Im(y)), \xi(\Im(ð), \Im(z))\} \\
= & \pi(\max \{\xi(\Im(\wp), \Im(x)), \xi(\Im(\hbar), \Im(y)), \xi(\Im(ð), \Im(z))\}) \\
& -\zeta \max \{\xi(\Im(\wp), \Im(x)), \xi(\Im(\hbar), \Im(y)), \xi(\Im(\Im), \Im(z))\} \\
= & \Lambda\left(\begin{array}{r}
\pi(\max \{\xi(\Im(\wp), \Im(x)), \xi(\Im(\hbar), \Im(y)), \xi(\Im(\Im), \Im(z))\}), \\
\zeta \max \{\xi(\Im(\wp), \Im(x)), \xi(\Im(\hbar), \Im(y)), \xi(\Im(\Im), \Im(z))\}
\end{array}\right)
\end{aligned}
$$

Thus, all the suppositions of Theorem 3.2 are contented and $(0,0,0)$ is a tripled coincidence common fixed point of $\Im$ and $\Re$.

Example 3.6 Assume that all data of Example 3.1 are validated except the mappings as follows: $\Im: \chi \rightarrow \chi$ and $\Xi: \chi^{3} \rightarrow \chi$ defined by

$$
\Im(\wp)=\frac{1}{10} \wp, \quad \Re(\wp, \hbar, \partial)=\frac{\wp-\hbar+ð}{40} .
$$

Then

$$
\begin{aligned}
(\Re(\wp, \hbar, ð), \Re(x, y, z)) & =\frac{\wp-\hbar+\partial}{80}+\frac{x-y+z}{80} \\
& =\frac{\wp+x}{80}-\frac{\hbar+y}{80}+\frac{\partial+z}{80}
\end{aligned}
$$




$$
\begin{aligned}
& \leq \frac{\wp+x}{80}+\frac{\hbar+y}{80}+\frac{\partial+z}{80} \\
& =\frac{1}{8}\left\{\left(\frac{\wp+x}{10}\right)+\left(\frac{\hbar+y}{10}\right)+\left(\frac{\partial+z}{10}\right)\right\} \\
& \leq \frac{3}{8} \max \left\{\frac{\wp+x}{10}, \frac{\hbar+y}{10}, \frac{\partial+z}{10}\right\} \\
& \leq \frac{3}{4} \times \frac{1}{2} \max \{\xi(\Im(\wp), \Im(x)), \xi(\Im(\hbar), \Im(y)), \xi(\Im(ð), \Im(z))\} \\
& =\frac{3}{4} \pi(\max \{\xi(\Im(\wp), \Im(x)), \xi(\Im(\hbar), \Im(y)), \xi(\Im(ð), \Im(z))\}) \\
& =\Lambda\left(\begin{array}{c}
\pi(\max \{\xi(\Im(\wp), \Im(x)), \xi(\Im(\hbar), \Im(y)), \xi(\Im(\Im), \Im(z))\}), \\
\zeta \max \{\xi(\Im(\wp), \Im(x)), \xi(\Im(\hbar), \Im(y)), \xi(\Im(\Im), \Im(z))\}
\end{array}\right) .
\end{aligned}
$$

Thus, all the suppositions of Theorem 3.2 are contented and $(0,0,0)$ is a tripled coincidence common fixed point of $\Im$ and $\Re$.

\section{Consequences of the main results}

This section is devoted to discussing some immediate consequences of the above theorems as follows:

If we devote $\Lambda(\lambda, \mu)=\lambda-\mu$ for all $\lambda, \mu \in \chi$ in Theorems 3.1 and 3.2, we get the following.

Corollary 4.1 Assume that $\Xi: \chi^{3} \rightarrow \chi$ and $\Theta: \chi \rightarrow \chi$ are two mappings on a POCML space $(\chi, \precsim, \xi)$ such that:

(i) $\Re\left(\chi^{3}\right) \subseteq \Im(\chi)$;

(ii) $\mathfrak{R}$ is continuous;

(iii) $\Im$ is continuous and commutes with $\Re$;

(iv) $\Re$ has a mixed $\mathfrak{\Im}$-monotone property;

(v) there are $\pi \in \Pi, \zeta \geq 0$, and $\Lambda \in \complement$ such that

$$
\begin{aligned}
\xi(\Re(\wp, \hbar, ð), \Re(x, y, z)) \leq & \pi(\max \{\xi(\Im(\wp), \Im(x)), \xi(\Im(\hbar), \Im(y)), \xi(\Im(ð), \Im(z))\}) \\
& -\zeta \max \{\xi(\Im(\wp), \Im(x)), \xi(\Im(\hbar), \Im(y)), \xi(\Im(ð), \Im(z))\}
\end{aligned}
$$

for any $\wp, \hbar, \precsim, x, y, z \in \chi$, for which $\Im(\wp) \precsim \Im(x), \Im(y) \precsim \Im(\hbar)$, and $\Im(ð) \precsim \Im(z)$. If there exist $\wp_{\circ}, \hbar_{\circ}, \partial_{\circ} \in \chi$ such that $\Im\left(\wp_{\circ}\right) \precsim \Re\left(\wp_{\circ}, \hbar_{\circ}, \partial_{\circ}\right), \Im\left(\hbar_{\circ}\right) \succsim \Re\left(\hbar_{\circ}, \wp_{\circ}, \partial_{\circ}\right)$, and $\Im\left(\varpi_{\circ}\right) \precsim$ $\Re\left(\Im_{0}, \hbar_{\circ}, \wp_{\circ}\right)$. Then $\Re$ and $\Im$ have a tripled coincidence point.

Corollary 4.2 Let $(\chi, \precsim, \xi)$ be a POCML space. Assume that $\Xi: \Upsilon \times \Upsilon \times \Upsilon \rightarrow \Upsilon$ and $\Theta: \Upsilon \rightarrow \Upsilon$ fulfill the following hypotheses:

(i) $\Re\left(\chi^{3}\right) \subseteq \Im(\chi)$;

(ii) $\Re$ has a mixed $\Im$-monotone property;

(iii) $(\Im(\chi), \xi)$ is a complete metric-like space and $\chi$ is obligated by the following assumptions:

(I) $l_{n} \precsim l$ if a nondecreasing sequence $l_{n} \rightarrow l, n \rightarrow+\infty$,

(II) $j_{n} \succsim j$ if a nonincreasing sequence $j_{n} \rightarrow j, n \rightarrow+\infty$;

(iv) $\Im$ is continuous and commutes with $\Re$; 
(v) there are $\pi \in \Pi, \zeta \geq 0$, and $\Lambda \in \complement$ such that

$$
\begin{aligned}
\xi(\Re(\wp, \hbar, ð), \Re(x, y, z)) \leq & \pi(\max \{\xi(\Im(\wp), \Im(x)), \xi(\Im(\hbar), \Im(y)), \xi(\Im(ð), \Im(z))\}) \\
& -\zeta \max \{\xi(\Im(\wp), \Im(x)), \xi(\Im(\hbar), \Im(y)), \xi(\Im(ð), \Im(z))\} .
\end{aligned}
$$

If there exist $\wp_{\circ}, \hbar_{\circ}, \partial_{\circ} \in \chi$ such that $\Im\left(\wp_{\circ}\right) \precsim \Re\left(\wp_{\circ}, \hbar_{\circ}, \widetilde{\partial}_{\circ}\right), \Im\left(\hbar_{\circ}\right) \succsim \Re\left(\hbar_{\circ}, \wp_{\circ}, \partial_{\circ}\right)$, and $\Im\left(\varpi_{\circ}\right) \precsim \Re\left(\varpi_{\circ}, \hbar_{\circ}, \wp_{\circ}\right)$, then $\Re$ and $\Im$ have a tripled coincidence point.

Corollary 4.3 Assume that $\Xi: \chi^{3} \rightarrow \chi$ and $\Theta: \chi \rightarrow \chi$ are two mappings on a POCML space $(\chi, \precsim, \xi)$ such that:

(i) $\Re\left(\chi^{3}\right) \subseteq \Im(\chi)$;

(ii) $\Re$ is continuous;

(iii) $\Im$ is continuous and commutes with $\Re$;

(iv) $\Re$ has a mixed $\Im$-monotone property;

(v) there are $\pi \in \Pi, \zeta \geq 0$, and $\Lambda \in \complement$ such that

$$
\begin{aligned}
\xi(\Re(\wp, \hbar, ð), \Re(x, y, z)) \leq & \pi\left(\frac{\xi(\Im(\wp), \Im(x))+\xi(\Im(\hbar), \Im(y))+\xi(\Im(ð), \Im(z))}{3}\right) \\
& -\zeta\left(\frac{\xi(\Im(\wp), \Im(x))+\xi(\Im(\hbar), \Im(y))+\xi(\Im(\Im), \Im(z))}{3}\right)
\end{aligned}
$$

for any $\wp, \hbar, ð, x, y, z \in \chi$, for which $\Im(\wp) \precsim \Im(x), \Im(y) \precsim \Im(\hbar)$, and $\Im(ð) \precsim \Im(z)$. If there exist $\wp_{\circ}, \hbar_{0}, \check{\partial}_{\circ} \in \chi$ such that $\Im\left(\wp_{\circ}\right) \precsim \Re\left(\wp_{0}, \hbar_{\circ}, \varpi_{\circ}\right), \Im\left(\hbar_{\circ}\right) \succsim \Re\left(\hbar_{\circ}, \wp_{\circ}, \partial_{\circ}\right)$, and $\Im\left(\varpi_{\circ}\right) \precsim$ $\Re\left(\Im_{\circ}, \hbar_{\circ}, \wp_{\circ}\right)$, then $\Re$ and $\Im$ have a tripled coincidence point.

Proof It is sufficient to note that

$$
\frac{\xi(\Im(\wp), \Im(x))+\xi(\Im(\hbar), \Im(y))+\xi(\Im(\Im), \Im(z))}{3} \leq \max \left\{\begin{array}{c}
\xi(\Im(\wp), \Im(x)), \\
\xi(\Im(\hbar), \Im(y)), \\
\xi(\Im(ð), \Im(z))
\end{array}\right\} .
$$

Since $\pi$ is nondecreasing, so we can apply Corollary 4.1.

Corollary 4.4 Let $(\chi, \precsim \xi)$ be a POCML space. Assume that $\Xi: \Upsilon \times \Upsilon \times \Upsilon \rightarrow \Upsilon$ and $\Theta: \Upsilon \rightarrow \Upsilon$ fulfill the following hypotheses:

(i) $\Re\left(\chi^{3}\right) \subseteq \Im(\chi)$;

(ii) $\Re$ has a mixed $\Im$-monotone property;

(iii) $(\Im(\chi), \xi)$ is a complete metric-like space and $\chi$ is obligated by the following assumptions:

(I) $l_{n} \precsim l$ if a nondecreasing sequence $l_{n} \rightarrow l, n \rightarrow+\infty$,

(II) $j_{n} \succsim j$ if a nonincreasing sequence $j_{n} \rightarrow j, n \rightarrow+\infty$;

(iv) $\Im$ is continuous and commutes with $\Re$;

(v) there are $\pi \in \Pi, \zeta \geq 0$, and $\Lambda \in \complement$ such that

$$
\begin{aligned}
\xi(\Re(\wp, \hbar, \Im), \Re(x, y, z)) \leq & \pi\left(\frac{\xi(\Im(\wp), \Im(x))+\xi(\Im(\hbar), \Im(y))+\xi(\Im(\Im), \Im(z))}{3}\right) \\
& -\zeta\left(\frac{\xi(\Im(\wp), \Im(x))+\xi(\Im(\hbar), \Im(y))+\xi(\Im(\Im), \Im(z))}{3}\right) .
\end{aligned}
$$


If there exist $\wp_{\circ}, \hbar_{\circ}, \check{\partial}_{\circ} \in \chi$ such that $\Im\left(\wp_{\circ}\right) \precsim \Re\left(\wp_{\circ}, \hbar_{\circ}, \check{\partial}_{\circ}\right), \Im\left(b_{\circ}\right) \succsim \Re\left(\hbar_{\circ}, \wp_{\circ}, \check{\partial}_{\circ}\right)$, and $\Im\left(\coprod_{\circ}\right) \precsim \Re\left(\coprod_{\circ}, \hbar_{\circ}, \wp_{\circ}\right)$, then $\Re$ and $\Im$ have a tripled coincidence point.

Proof It is enough to remark

$$
\frac{\xi(\Im(\wp), \Im(x))+\xi(\Im(\hbar), \Im(y))+\xi(\Im(\Im), \Im(z))}{3} \leq \max \left\{\begin{array}{l}
\xi(\Im(\wp), \Im(x)), \\
\xi(\Im(\hbar), \Im(y)), \\
\xi(\Im(ð), \Im(z))
\end{array}\right\} .
$$

So we can use Corollary 4.2 to complete the required.

Customizing $\Lambda(\lambda, \mu)=\varrho \lambda, 0<\varrho<1$ in Theorems 3.1 and 3.2, we have the following.

Corollary 4.5 Assume that $\Xi: \chi^{3} \rightarrow \chi$ and $\Theta: \chi \rightarrow \chi$ are two mappings on a POCML space $(\chi, \precsim, \xi)$ such that:

(i) $\Re\left(\chi^{3}\right) \subseteq \Im(\chi)$;

(ii) $\Re$ is continuous;

(iii) $\Im$ is continuous and commutes with $\Re$;

(iv) $\Re$ has a mixed $\Im$-monotone property;

(v) there are $\pi \in \Pi, \zeta \geq 0$, and $\Lambda \in \complement$ such that

$$
\xi(\Re(\wp, \hbar, \partial), \Re(x, y, z)) \leq \varrho \pi(\max \{\xi(\Im(a), \Im(x)), \xi(\Im(b), \Im(y)), \xi(\Im(c), \Im(z))\})
$$

for any $\wp, \hbar, ð, x, y, z \in \chi$, for which $\Im(\wp) \precsim \Im(x), \Im(y) \precsim \Im(\hbar)$, and $\Im(ð) \precsim \Im(z)$. If there exist $\wp_{\circ}, \hbar_{\circ}, \partial_{\circ} \in \chi$ such that $\Im\left(\wp_{\circ}\right) \precsim \Re\left(\wp_{0}, \hbar_{0}, \check{\partial}_{\circ}\right), \Im\left(\hbar_{\circ}\right) \succsim \Re\left(\hbar_{\circ}, \wp_{0}, \varpi_{\circ}\right)$, and $\Im\left(\varpi_{\circ}\right) \precsim$ $\Re\left(\Im_{\circ}, \hbar_{\circ}, \wp_{\circ}\right)$, then $\Re$ and $\Im$ have a tripled coincidence point.

Corollary 4.6 Let $(\chi, \precsim, \xi)$ be a POCML space. Assume that $\Xi: \Upsilon \times \Upsilon \times \Upsilon \rightarrow \Upsilon$ and $\Theta: \Upsilon \rightarrow \Upsilon$ fulfill the following hypotheses:

(i) $\Re\left(\chi^{3}\right) \subseteq \Im(\chi)$;

(ii) $\Re$ has a mixed $\Im$-monotone property;

(iii) $(\Im(\chi), \xi)$ is a complete metric-like space and $\chi$ is obligated by the following assumptions:

(I) $l_{n} \precsim l$ if a nondecreasing sequence $l_{n} \rightarrow l, n \rightarrow+\infty$,

(II) $j_{n} \succsim j$ if a nonincreasing sequence $j_{n} \rightarrow j, n \rightarrow+\infty$;

(iv) $\Im$ is continuous and commutes with $\Re$;

(v) there are $\pi \in \Pi, \zeta \geq 0$, and $\Lambda \in \complement$ such that

$$
\xi(\Re(\wp, \hbar, ð), \Re(x, y, z)) \leq \varrho \pi(\max \{\xi(\Im(\wp), \Im(x)), \xi(\Im(\hbar), \Im(y)), \xi(\Im(ð), \Im(z))\}) .
$$

If there exist $\wp_{\circ}, \hbar_{\circ}, \partial_{\circ} \in \chi$ such that $\Im\left(\wp_{\circ}\right) \precsim \Re\left(\wp_{\circ}, \hbar_{\circ}, \partial_{\circ}\right), \Im\left(\hbar_{\circ}\right) \succsim \Re\left(\hbar_{\circ}, \wp_{\circ}, \check{\partial}_{\circ}\right)$, and $\Im\left(\Im_{\circ}\right) \precsim \Re\left(\Im_{\circ}, \hbar_{\circ}, \wp_{\circ}\right)$, then $\Re$ and $\Im$ have a tripled coincidence point.

Now, if we choose $\mathfrak{\Im}=I_{\chi}$ (where $I_{\chi}$ is the identity mapping) and replace a mixedmonotone property with a monotone-increasing one in Theorem 3.1, we get the following important result. 
Corollary 4.7 Let $(\Re, \precsim, \xi)$ be a POCML space. Suppose that $\Re: \chi^{3} \rightarrow \chi$ is a mapping such that:

(i) $\Re$ is continuous;

(ii) $\Re$ is nondecreasing with respect to $\precsim$;

(iii) there exist three elements $\wp_{\circ}, \hbar_{\circ}, c_{\circ} \in \chi$ such that $\wp_{\circ} \precsim \Re\left(\wp_{\circ}, \hbar_{\circ}, \partial_{\circ}\right)$,

$$
\hbar_{\circ} \succsim \Re\left(\hbar_{\circ}, \wp_{\circ}, \hbar_{\circ}\right) \text {, and } c_{\circ} \precsim \Re\left(c_{\circ}, \hbar_{\circ}, \wp_{\circ}\right) ;
$$

(iv) there are $\pi \in \Pi, \zeta \geq 0$, and $\Lambda \in \complement$ such that

$$
\begin{aligned}
& \xi(\Re(\wp, \hbar, ð), \Re(x, y, z)) \\
& \quad \leq \Lambda(\pi(\max \{\xi(\wp, x), \xi(\hbar, y), \xi(ð, z)\}), \zeta \max \{\xi(\wp, x), \xi(\hbar, y), \xi(ð, z)\})
\end{aligned}
$$

for any $\wp, \hbar, \partial, x, y, z \in \chi$, and for which $\wp \precsim x, y \precsim \hbar$, and $\precsim \precsim z$. Then there is a tripled coincidence point of $\Re$.

\section{Applications}

This part is considered as the mainstay of this paper because it indicates the applications that contribute to solving some nonlinear integral systems that attract many readers and researchers and show the importance of the fixed point theory in many areas.

\subsection{Some contributions of integral type}

Let $\Omega$ be a class of functions $\varpi:[0,+\infty) \rightarrow[0,+\infty)$ fulfilling the following postulates:

(i) For each compact subset of $[0,+\infty)$, $\varpi$ is a positive Lebesgue integrable mapping;

(ii) $\int_{0}^{\epsilon} \varpi(\ell) d \ell>0$ for all $\epsilon>0$.

Corollary 5.1 Let $\Lambda(\lambda, \mu)=\lambda-\mu$. Exchange stipulation (1) of Theorem 3.1 by the formula

$$
\int_{0}^{\xi(\Re(\wp, \hbar, \widetilde{\varnothing}), \Re(x, y, z))} \varpi(\ell) d \ell \leq \int_{0}^{\pi(\varphi(\wp, x, \hbar, y, y, \widetilde{\partial}, z))-\zeta \varphi(\wp, x, \hbar, y, \widetilde{\partial}, z)} \varpi(\ell) d \ell
$$

for all $\varpi \in \Omega$, where $\varphi(\wp, x, \hbar, y, \precsim, z)=\max \{\xi(\Im(\wp), \Im(x)), \xi(\Im(\hbar), \Im(y)), \xi(\Im(\Im), \Im(z))\}$. If other hypotheses of Theorem 3.1 are fulfilled, then there is a tripled coincidence point of the mentioned mappings.

Proof Suppose the function $\Upsilon(\wp)=\int_{0}^{\wp} \varpi(\ell) d \ell$, then (33) becomes

$$
\Upsilon(\xi(\Re(\wp, \hbar, ð), \Re(x, y, z))) \leq \Upsilon \pi(\varphi(\wp, x, \hbar, y, \partial, z))-\Upsilon(\zeta \varphi(\wp, x, \hbar, y, \partial, z)) .
$$

Letting $\pi_{1}=\Upsilon \circ \pi$, we have $\pi_{1} \in \Pi$, since $\Upsilon \zeta \geq 0$, then the proof is quickly completed from Theorem 3.1.

Corollary 5.2 Let $\Lambda(\lambda, \mu)=\lambda-\mu$. Exchange stipulation (1) of Theorem 3.1 by the formula

$$
\begin{aligned}
& \int_{0}^{\xi(\Re(\wp, \hbar, \widetilde{\Im}), \Re(x, y, z))} \varpi(\ell) d \ell \\
& \quad \leq \pi\left(\int_{0}^{\varphi(\wp, x, \hbar, y, \widetilde{\partial}, z)} \varpi(\ell) d \ell\right)-\zeta \int_{0}^{\varphi(\wp, x, \hbar, y, \widetilde{\partial}, z)} \varpi(\ell) d \ell
\end{aligned}
$$


for each $\varpi \in \Omega$, If other hypotheses of Theorem 3.1 are fulfilled, then there is a tripled coincidence point of the mentioned mappings.

Proof As in Corollary 5.1, define the function $\Upsilon(\wp)=\int_{0}^{\wp} \varpi(\ell) d \ell$, then (34) is

$$
\begin{aligned}
& \Upsilon(\xi(\Im(\wp, \hbar, \precsim), \Re(x, y, z))) \\
& \quad \leq \pi(\Upsilon(\varphi(\wp, x, \hbar, y, ð, z)))-\zeta \Upsilon(\varphi(\wp, x, \hbar, y, \partial, z)) .
\end{aligned}
$$

Putting $\pi_{2}=\pi \circ \Upsilon$, we get $\pi_{2} \in \Pi$, since $\zeta \Upsilon \geq 0$, then the proof is quickly completed from Theorem 3.1.

In the same line of [51], let a fixed number $\nabla \in \mathbb{N}$. Suppose that $\left\{\varpi_{j}\right\}_{1 \leq j \leq \nabla}$ is a collection of $\nabla$ functions which belong to $\Omega$. For each $\ell \geq 0$, we define

$$
\begin{aligned}
& J_{1}(\ell)=\int_{0}^{\ell} \varpi_{1}(\rho) d \rho, \\
& J_{2}(\ell)=\int_{0}^{J_{1}(\ell)} \varpi_{2}(\rho) d \rho=\int_{0}^{\int_{0}^{\ell} \varpi_{1}(\rho) d \rho,} \varpi_{2}(\rho) d \rho, \\
& J_{3}(\ell)=\int_{0}^{J_{2}(\ell)} \varpi_{3}(\rho) d \rho=\int_{0}^{\int_{0}^{\int_{0}^{\ell} \varpi_{1}(\rho) d \rho,} \varpi_{2}(\rho) d \rho} \varpi_{3}(\rho) d \rho, \\
& \ldots \\
& J_{\nabla}(\ell)=\int_{0}^{J_{(\nabla-1)}(\ell)} \varpi_{\nabla}(\rho) d \rho .
\end{aligned}
$$

We have the following consequence.

Corollary 5.3 Let $\Lambda(\lambda, \mu)=\lambda-\mu$. Replace inequality (1) of Theorem 3.1 by the the following assumption: There is $\varpi \in \Omega$ such that

$$
J_{\nabla}(\xi(\Re(\wp, \hbar, ð), \Re(x, y, z))) \leq \pi\left(J_{\nabla}(\varphi(\wp, x, \hbar, y, ð, z))-\zeta J_{\nabla}(\varphi(\wp, x, \hbar, y, ð, z) .\right.
$$

If the remaining conditions of Theorem 3.1 are true, then there is a tripled coincidence point of $\Re$ and $\Im$.

Proof Specify $\pi_{3}=\pi \circ J_{\nabla}$ and $\pi_{4}=\zeta \circ J_{\nabla}$, then inequality (35) takes the form

$$
J_{\nabla}(\xi(\Re(\wp, \hbar, ð), \Re(x, y, z))) \leq \pi_{3}\left(\varphi(\wp, x, \hbar, y, ð, z)-\psi_{4}(\varphi(\wp, x, \hbar, y, ð, z) .\right.
$$

Applying Theorem 3.1, we obtain the desired result because $\pi_{2} \in \Pi$ and $\pi_{4}=\zeta J_{\nabla} \geq 0$.

\subsection{Solve a system of nonlinear integral equations}

Let $\Omega$ be a class functions $\omega:[0,+\infty) \rightarrow[0,+\infty)$ such that $\omega$ is increasing and there exist $\pi \in \Pi, \zeta \geq 0$, and $\Lambda \in \complement$ such that $\omega(\mu)=\frac{1}{3} \Lambda(\pi(\mu), \zeta \mu)$ for all $\mu \in[0,+\infty)$. 
Consider the following problem:

$$
\begin{aligned}
\wp(v)= & \varphi(v)+\int_{p}^{q}\left(r_{1}(v, \rho)+r_{2}(v, \rho)+r_{3}(v, \rho)\right) \\
& \times\left[p_{1}(\rho, \wp(\rho))+p_{2}(\rho, \hbar(\rho))+p_{3}(\rho, \partial(\rho))\right] d \rho
\end{aligned}
$$

for all $v \in[p, q]$. We postulate that the following assumptions hold:

(i) $\varphi:[p, q] \rightarrow \mathbb{R}$ is continuous;

(ii) $p_{i}, r_{i}(i=1,2,3):[p, q] \times \mathbb{R} \rightarrow \mathbb{R}$ are continuous;

(iii) For all $\wp, \partial \in \mathbb{R}$, there are $\varkappa, \tau, \sigma$ such that

$$
\begin{aligned}
& 0 \leq p_{1}(\rho, \wp)-p_{1}(\rho, ð) \leq \varkappa \omega(\wp-ð), \\
& 0 \leq p_{2}(\rho, \wp)-p_{2}(\rho, ð) \leq \tau \omega(\wp-ð),
\end{aligned}
$$

and

$$
0 \leq p_{3}(\rho, \wp)-p_{3}(\rho, c) \leq \sigma \omega(\wp-\succsim) ;
$$

(iv) We assume that

$$
\max \{\varkappa, \tau, \sigma\}\left(\sup _{t \in[p, q]} \int_{p}^{q}\left[r_{1}(v, \rho)+r_{2}(v, \rho)+r_{3}(v, \rho)\right] d \rho\right) \leq 1
$$

(v) There are continuous functions $\alpha, \beta, \gamma:[p, q] \rightarrow \mathbb{R}$ such that

$$
\begin{aligned}
\alpha(v) \leq & \int_{p}^{q} r_{1}(v, \rho)\left[p_{1}(\rho, \alpha(\rho))+p_{2}(\rho, \beta(\rho))+p_{3}(\rho, \gamma(\rho))\right] d \rho \\
& +\int_{p}^{q} r_{2}(v, \rho)\left[p_{1}(\rho, \delta(\rho))+p_{2}(\rho, \eta(\rho))+p_{3}(\rho, \delta(\rho))\right] d \rho \\
& +\int_{p}^{q} r_{3}(v, \rho)\left[p_{1}(\rho, \gamma(\rho))+p_{2}(\rho, \beta(\rho))+p_{3}(\rho, \alpha(\rho))\right] d \rho, \\
\beta(v) \leq & \int_{p}^{q} r_{1}(v, \rho)\left[p_{1}(\rho, \beta(\rho))+p_{2}(\rho, \eta(\rho))+p_{3}(\rho, \beta(\rho))\right] d \rho \\
& +\int_{p}^{q} r_{2}(v, \rho)\left[p_{1}(\rho, \gamma(\rho))+p_{2}(\rho, \beta(\rho))+p_{3}(\rho, \alpha(\rho))\right] d \rho \\
& +\int_{p}^{q} r_{3}(v, \rho)\left[p_{1}(\rho, \alpha(\rho))+p_{2}(\rho, \beta(\rho))+p_{3}(\rho, \gamma(\rho))\right],
\end{aligned}
$$

and

$$
\begin{aligned}
\gamma(v) \leq & \int_{p}^{q} r_{1}(v, \rho)\left[p_{1}(\rho, \gamma(\rho))+p_{2}(\rho, \beta(\rho))+p_{3}(\rho, \alpha(\rho))\right] d \rho \\
& +\int_{p}^{q} r_{2}(v, \rho)\left[p_{1}(\rho, \alpha(\rho))+p_{2}(\rho, \beta(\rho))+p_{3}(\rho, \gamma(\rho))\right] d \rho \\
& +\int_{p}^{q} r_{3}(v, \rho)\left[p_{1}(\rho, \beta(\rho))+p_{2}(\rho, \alpha(\rho))+p_{3}(\rho, \beta(\rho))\right] d \rho .
\end{aligned}
$$


Let $\chi=C([p, q], \mathbb{R})$ be the set of real continuous functions on $[p, q]$ endowed with

$$
\xi(\wp, \hbar)=\|\wp-\hbar\|_{\infty}=\sup _{v \in[p, q]}\{|\wp(v)-\hbar(v)|\}
$$

for all $\wp, \hbar \in \chi$. Then the pair $(\chi, \xi)$ is a complete metric-like space. We endow $\chi$ with the partial order $\precsim$ as follows:

$$
\wp \precsim \hbar \Leftrightarrow \wp(v) \leq \hbar(v), \quad \forall v \in[p, q] .
$$

Subsequently, $(\chi, \precsim, \xi)$ is a POCML space if $\wp \precsim x, y \precsim \hbar$, and $\precsim \precsim z$ whenever $\wp(v) \leq x(v)$, $y(v) \leq \hbar(v)$, and $ð(v) \leq z(v)$ for all $\wp, \hbar, ð, x, y, z \in \chi$ and $v \in[p, q]$.

Now, we can state and prove our main theorem of this section.

Theorem 5.4 Under hypotheses (i)-(v), problem (36) has a solution in $\chi^{3}$, where $\chi=$ $C([p, q], \mathbb{R})$.

Proof Define an operator $\Re: \chi^{3} \rightarrow \chi$ by

$$
\begin{aligned}
\Re(\wp, \hbar, \partial)(v) & \\
= & (v)+\int_{p}^{q} r_{1}(v, \rho)\left[p_{1}(\rho, \wp(\rho))+p_{2}(\rho, \hbar(\rho))+p_{3}(\rho, \partial(\rho))\right] d \rho \\
& +\int_{p}^{q} r_{2}(v, \rho)\left[p_{1}(\rho, \hbar(\rho))+p_{2}(\rho, \wp(\rho))+p_{3}(\rho, \hbar(\rho))\right] d \rho \\
& +\int_{p}^{q} r_{3}(v, \rho)\left[p_{1}(\rho, \partial(\rho))+p_{2}(\rho, \hbar(\rho))+p_{3}(\rho, \wp(\rho))\right] d \rho
\end{aligned}
$$

for all $v \in[p, q]$ and $\wp, \hbar, \partial \in \chi$. It is clear that if the mapping $\Xi$ has a tripled coincidence point in $\Upsilon=C([p, q], \mathbb{R})$, then it is a solution of problem (36).

Now, we shall prove the increasing property of the mapping $\Xi$ with $\wp_{1} \precsim \wp_{2}$, so $\wp_{1}(\nu) \leq$ $\wp_{2}(v)$ for all $v \in[p, q]$, we get

$$
\begin{aligned}
\Re\left(\wp_{1}, \hbar, \partial\right)(v)-\Xi\left(\wp_{2}, \hbar, \partial\right)(v) & \int_{p}^{q} r_{1}(v, \rho)\left[p_{1}\left(\rho, \wp_{1}(\rho)\right)+p_{2}(\rho, \hbar(\rho))+p_{3}(\rho, \partial(\rho))\right] d \rho \\
& +\int_{p}^{q} r_{2}(v, \rho)\left[p_{1}(\rho, \hbar(\rho))+p_{2}\left(\rho, \wp_{1}(\rho)\right)+p_{3}(\rho, \hbar(\rho))\right] d \rho \\
& +\int_{p}^{q} r_{3}(v, \rho)\left[p_{1}(\rho, \partial(\rho))+p_{2}(\rho, \hbar(\rho))+p_{3}\left(\rho, \wp_{1}(\rho)\right)\right] d \rho \\
& -\int_{p}^{q} r_{1}(v, \rho)\left[p_{1}\left(\rho, \wp_{2}(\rho)\right)+p_{2}(\rho, \hbar(\rho))+p_{3}(\rho, \partial(\rho))\right] d \rho \\
& -\int_{p}^{q} r_{2}(v, \rho)\left[p_{1}(\rho, \hbar(\rho))+p_{2}\left(\rho, \wp_{2}(\rho)\right)+p_{3}(\rho, \hbar(\rho))\right] d \rho \\
& -\int_{p}^{q} r_{3}(v, \rho)\left[p_{1}(\rho, \partial(\rho))+p_{2}(\rho, \hbar(\rho))+p_{3}\left(\rho, \wp_{2}(\rho)\right)\right] d \rho
\end{aligned}
$$




$$
\begin{aligned}
= & \int_{p}^{q} r_{1}(v, \rho)\left[p_{1}\left(\rho, \wp_{1}(\rho)\right)-p_{1}\left(\rho, \wp_{2}(\rho)\right)\right] d \rho \\
& +\int_{p}^{q} r_{2}(v, \rho)\left[p_{2}\left(\rho, \wp_{1}(\rho)\right)-p_{2}\left(\rho, \wp_{2}(\rho)\right)\right] d \rho \\
& +\int_{p}^{q} r_{3}(v, \rho)\left[p_{3}\left(\rho, \wp_{1}(\rho)\right)-p_{3}\left(\rho, \wp_{2}(\rho)\right)\right] d \rho \\
\leq & 0 .
\end{aligned}
$$

Hence, $\Re\left(\wp_{1}, \hbar, ð\right)(v) \leq \Re\left(\wp_{2}, \hbar, \partial\right)(v)$ for all $v \in[p, q]$. Subsequently, $\Re\left(\wp_{1}, \hbar, ð\right) \precsim$ $\Re\left(\wp_{2}, \hbar, ð\right)$.

Again, if $\hbar_{1} \precsim \hbar_{2}$, so $\hbar_{1}(v) \leq \hbar_{2}(v)$ for all $v \in[p, q]$, we can get

$$
\begin{aligned}
\Re(\wp, & \left.\hbar_{1}, \partial\right)(v)-\Re\left(\wp, \hbar_{2}, \partial\right)(v) \\
= & \int_{p}^{q} r_{1}(v, \rho)\left[p_{1}(\rho, \wp(\rho))+p_{2}\left(\rho, \hbar_{1}(\rho)\right)+p_{3}(\rho, \partial(\rho))\right] d \rho \\
& +\int_{p}^{q} r_{2}(v, \rho)\left[p_{1}\left(\rho, \hbar_{1}(\rho)\right)+p_{2}(\rho, \wp(\rho))+p_{3}\left(\rho, \hbar_{1}(\rho)\right)\right] d \rho \\
& +\int_{p}^{q} r_{3}(v, \rho)\left[p_{1}(\rho, \partial(\rho))+p_{2}\left(\rho, \hbar_{1}(\rho)\right)+p_{3}(\rho, \wp(\rho))\right] d \rho \\
& -\int_{p}^{q} r_{1}(v, \rho)\left[p_{1}(\rho, \wp(\rho))+p_{2}\left(\rho, \hbar_{2}(\rho)\right)+p_{3}(\rho, \partial(\rho))\right] d \rho \\
& \quad-\int_{p}^{q} r_{2}(v, \rho)\left[p_{1}\left(\rho, \hbar_{2}(\rho)\right)+p_{2}(\rho, \wp(\rho))+p_{3}\left(\rho, \hbar_{2}(\rho)\right)\right] d \rho \\
& -\int_{p}^{q} r_{3}(v, \rho)\left[p_{1}(\rho, \partial(\rho))+p_{2}\left(\rho, \hbar_{2}(\rho)\right)+p_{3}(\rho, \wp(\rho))\right] d \rho \\
= & \int_{p}^{q} r_{1}(v, \rho)\left[p_{2}\left(\rho, \hbar_{1}(\rho)\right)-p_{2}\left(\rho, \hbar_{2}(\rho)\right)\right] d \rho \\
& +\int_{p}^{q} r_{2}(v, \rho)\left[\left\{p_{1}\left(\rho, \hbar_{1}(\rho)\right)-p_{1}\left(\rho, \hbar_{2}(\rho)\right)\right\}\right] d \rho \\
\left.+\left\{p_{3}\left(\rho, \hbar_{1}(\rho)\right)-p_{3}\left(\rho, \hbar_{2}(\rho)\right)\right\}\right] & \\
& +\int_{p}^{q} r_{3}(v, \rho)\left[p_{2}\left(\rho, \hbar_{1}(\rho)\right)-p_{2}\left(\rho, \hbar_{2}(\rho)\right)\right] d \rho \\
\leq & 0 .
\end{aligned}
$$

So, $\Re\left(\wp, \hbar_{1}, \widetilde{\partial}\right) \leq \Re\left(\wp, \hbar_{2}, \widetilde{\partial}\right)(v)$ for all $v \in[p, q]$. Hence $\Re\left(\wp, \hbar_{1}, ð\right) \precsim \Re\left(\wp, \hbar_{2}, \widetilde{\partial}\right)$. In the same manner, we can write $\Re\left(\wp, \hbar, \partial_{1}\right) \precsim \Re\left(\wp_{2}, \hbar, \partial_{2}\right)$ if $\partial_{1} \precsim \partial_{2}$. From the above inequalities, we observe that the mapping $\Re$ is increasing with respect to the variables $\wp, \hbar$, and $ð$.

Finally, we shall verify contractive condition (32) of Corollary 4.7 for all $\wp, \hbar, ð, x, y, z \in \chi$ such that $\wp \precsim x, y \precsim \hbar$, and $\precsim \precsim z$,

$$
\begin{aligned}
& \xi(\Re(\wp, \hbar, ð), \Re(x, y, z)) \\
& \quad=\sup _{v \in[p, q]}\{|\Re(\wp, \hbar, ð)(v)-\Re(x, y, z)(v)|\}
\end{aligned}
$$




$$
\begin{aligned}
& =\sup _{\nu \in[p, q]}\left\{\begin{array}{l}
\mid \int_{p}^{q} r_{1}(\nu, \rho)\left[p_{1}(\rho, \wp(\rho))+p_{2}(\rho, \hbar(\rho))+p_{3}(\rho, \precsim(\rho))\right] d \rho \\
+\int_{p}^{q} r_{2}(\nu, \rho)\left[p_{1}(\rho, \hbar(\rho))+p_{2}(\rho, \wp(\rho))+p_{3}(\rho, \hbar(\rho))\right] d \rho \\
+\int_{p}^{q} r_{3}(\nu, \rho)\left[p_{1}(\rho, \precsim(\rho))+p_{2}(\rho, \hbar(\rho))+p_{3}(\rho, \wp(\rho))\right] d \rho \\
-\int_{p}^{q} r_{1}(\nu, \rho)\left[p_{1}(\rho, x(\rho))+p_{2}(\rho, y(\rho))+p_{3}(\rho, z(\rho))\right] d \rho \\
-\int_{p}^{q} r_{2}(\nu, \rho)\left[p_{1}(\rho, y(\rho))+p_{2}(\rho, x(\rho))+p_{3}(\rho, y(\rho))\right] d \rho \\
-\int_{p}^{q} r_{3}(\nu, \rho)\left[p_{1}(\rho, z(\rho))+p_{2}(\rho, y(\rho))+p_{3}(\rho, x(\rho))\right] d \rho \mid
\end{array}\right\}
\end{aligned}
$$

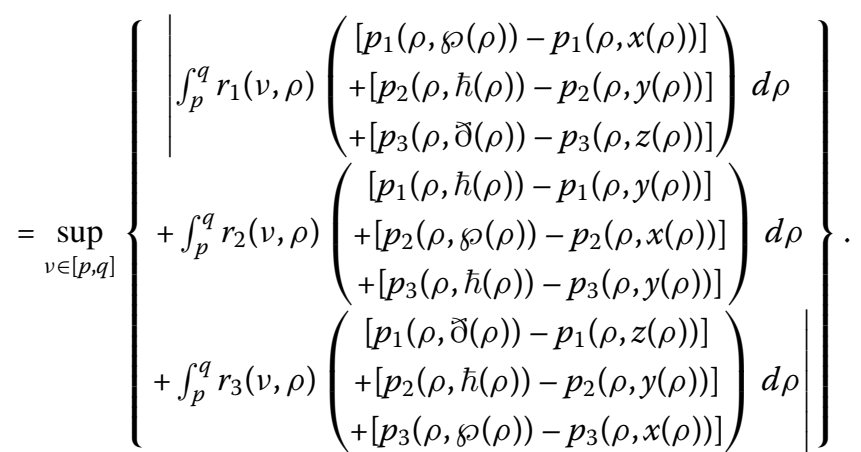

Applying hypothesis (iii), we get

$$
\begin{aligned}
& \xi(\Re(\wp, \hbar, \partial), \Re(x, y, z)) \\
& \leq \sup _{\nu \in[p, q]}\left\{\begin{array}{c}
\mid \int_{p}^{q} r_{1}(\nu, \rho)[\varkappa \omega(\wp(\rho)-x(\rho))+\tau \omega(\hbar(\rho)-y(\rho))+\sigma \omega(ð(\rho)-z(\rho))] d \rho \\
+\int_{p}^{q} r_{2}(\nu, \rho)[\varkappa \omega(\hbar(\rho)-y(\rho))+\tau \omega(\wp(\rho)-x(\rho))+\sigma \omega(\hbar(\rho)-y(\rho))] d \rho \\
+\int_{p}^{q} r_{3}(\nu, \rho)[\varkappa \omega(\partial(\rho)-z(\rho))+\tau \omega(\hbar(\rho)-y(\rho))+\sigma \omega(\wp(\rho)-x(\rho))] d \rho \mid
\end{array}\right\} \\
& \leq \max \{\varkappa, \tau, \sigma\} \\
& \quad \times \sup _{\nu \in[p, q]}\left\{\begin{array}{c}
\int_{p}^{q}\left(r_{1}(\nu, \rho)+r_{2}(\nu, \rho)+r_{3}(\nu, \rho)\right) \times \\
{[\omega(|\wp(\rho)-x(\rho)|)+\omega(|\hbar(\rho)-y(\rho)|)+\omega(|\partial(\rho)-z(\rho)|)] d \rho}
\end{array}\right\} .
\end{aligned}
$$

By the characterizations of the function $\omega$ and the distance $\xi$, one can write, for all $\rho \in$ $[p, q]$,

$$
\begin{aligned}
& \omega(|\wp(\rho)-x(\rho)|) \leq \omega \xi(\wp, x), \\
& \omega(|\hbar(\rho)-y(\rho)|) \leq \omega \xi(\hbar, y), \\
& \omega(|\varnothing(\rho)-z(\rho)|) \leq \omega \xi(ð, z) .
\end{aligned}
$$

It follows from (37), (38) and assumption (iv) that

$$
\begin{aligned}
\xi(\Re(\wp, \hbar, ð), \Re(x, y, z)) \\
\leq \quad \max \{\varkappa, \tau, \sigma\} \times(\omega \xi(\wp, x)+\omega \xi(\hbar, y)+\omega \xi(ð, z)) \\
\quad \times\left(\sup _{\nu \in[p, q]} \int_{p}^{q}\left(r_{1}(v, \rho)+r_{2}(v, \rho)+r_{3}(\nu, \rho)\right) d \rho\right) \\
\leq \omega \xi(\wp, x)+\omega \xi(\hbar, y)+\omega \xi(ð, z) \\
\leq 3 \omega(\max \{\xi(\wp, x), \xi(\hbar, y), \xi(\hbar, y)\})
\end{aligned}
$$




$$
\begin{aligned}
& \leq 3 \times \frac{1}{3} \Lambda(\pi(\max \{\xi(\wp, x), \xi(\hbar, y), \xi(\hbar, y)\}), \zeta \max \{\xi(\wp, x), \xi(\hbar, y), \xi(\hbar, y)\}) \\
& =\Lambda(\pi(\max \{\xi(\wp, x), \xi(\hbar, y), \xi(\hbar, y)\}), \zeta \max \{\xi(\wp, x), \xi(\hbar, y), \xi(\hbar, y)\}) .
\end{aligned}
$$

Also, condition (v) tells us $\alpha(v) \leq \Re(\alpha, \beta, \gamma)(v), \beta(v) \geq \Re(\beta, \alpha, \beta)(v)$, and $\gamma(v) \leq$ $\Re(\gamma, \beta, \alpha)(v)$ for all $v \in[p, q]$. This yields $\alpha \precsim \Re(\alpha, \beta, \gamma), \beta \succsim \Re(\beta, \alpha, \beta, \gamma)$, and $\gamma \precsim$ $\Re(\gamma, \beta, \alpha)$. Applying Corollary 4.7, we deduce the existence solution of problem (36).

\section{Acknowledgements}

The authors are thankful to the editor for their valuable comments and suggestions.

\section{Funding}

This work was supported in part by the Basque Government under Grant IT1207-19.

\section{Availability of data and materials}

The data used to support the findings of this study are available from the corresponding author upon request.

\section{Competing interests}

The authors declare that they have no competing interests concerning the publication of this article.

\section{Authors' contributions}

All authors contributed equally and significantly in writing this article. All authors read and approved the final manuscript.

\section{Author details}

'Department of Mathematics, Faculty of Science, Sohag University, Mohamed Farid-Elmeniawy, 82524 Sohag, Egypt.

${ }^{2}$ Institute of Research and Development of Processes, University of the Basque Country, 48940 Leioa (Bizkaia), Spain.

\section{Publisher's Note}

Springer Nature remains neutral with regard to jurisdictional claims in published maps and institutional affiliations.

Received: 1 May 2020 Accepted: 7 August 2020 Published online: 17 August 2020

\section{References}

1. Fredholm, E.I.: Sur une classe d'equations fonctionnelles. Acta Math. 27, 365-390 (1903)

2. Rus, M.D.: A note on the existence of positive solution of Fredholm integral equations. Fixed Point Theory 5, 369-377 (2004)

3. Berenguer, M.I., Munoz, M.V.F., Guillem, A.I.G., Galan, M.R.: Numerical treatment of fixed point applied to the nonlinear Fredholm integral equation. Fixed Point Theory Appl. 2009, 735638 (2009)

4. Hammad, H.A., De la Sen, M.: A solution of Fredholm integral equation by using the cyclic $\eta_{s}^{q}$-rational contractive mappings technique in $b$-metric-like spaces. Symmetry 11, 1184 (2019)

5. Ameer, E., Aydi, H., Arshad, M., De la Sen, M.: Hybrid Ćirić type graphic $(\Upsilon, \Lambda)$-contraction mappings with applications to electric circuit and fractional differential equations. Symmetry 12(3), 467 (2020)

6. Bhaskar, T.G., Lakshmikantham, V.: Fixed point theorems in partially ordered metric spaces and applications, Nonlinear Anal. TMA 65, 1379-1393 (2006)

7. Abbas, M., Khan, M.A., Radenović, S.: Common coupled fixed point theorems in cone metric spaces for $w$-compatible mappings. Appl. Math. Comput. 217, 195-202 (2010)

8. Aydi, H., Postolache, M., Shatanawi, W.: Coupled fixed point results for $(\psi, \phi)$-weakly contractive mappings in ordered G-metric spaces. Comput. Math. Appl. 63, 298-309 (2012)

9. Berinde, V:: Coupled fixed point theorems for contractive mixed monotone mappings in partially ordered metric spaces. Nonlinear Anal. 75, 3218-3228 (2012)

10. Choudhury, B.S., Maity, P.: Coupled fixed point results in generalized metric spaces. Math. Comput. Model. 54, 73-79 (2011)

11. Cirić, L., Damjanović, B., Jeli, M., Samet, B.: Coupled fixed point theorems for generalized Mizoguchi-Takahashi contraction and applications to ordinary differential equations. Fixed Point Theory Appl. 2012, Article ID 51 (2012)

12. Ding, H.S., Li, L., Radenovic, S.: Coupled coincidence point theorems for generalized nonlinear contraction in partially ordered metric spaces. Fixed Point Theory Appl. 2012, Article ID 96 (2012)

13. Hammad, H.A., De la Sen, M.: A coupled fixed point technique for solving coupled systems of functional and nonlinear integral equations. Mathematics 7, 634 (2019)

14. Luong, N.V., Thuan, N.X.: Coupled fixed points in partially ordered metric spaces and application. Nonlinear Anal. 74 983-992 (2011)

15. Razani, A., Parvaneh, V.: Coupled coincidence point results for $(\psi, \alpha, \beta)$-weak contractions in partially ordered metric spaces. J. Appl. Math. 2012, Article ID 496103 (2012)

16. Shatanawi, W.: Coupled fixed point theorems in generalized metric spaces. Hacet. J. Math. Stat. 40, 441-447 (2011)

17. Sintunavarat, W., Cho, Y.J., Kumam, P.: Coupled coincidence point theorems for contractions without commutative condition in intuitionistic fuzzy normed spaces. Fixed Point Theory Appl. 2011, Article ID 81 (2011)

18. Sintunavarat, W., Cho, Y.J., Kumam, P.: Coupled fixed point theorems for weak contraction mapping under $F$-invariant set. Abstr. Appl. Anal. 2012, Article ID 324874 (2012) 
19. Karapınar, E., Kumam, P., Sintunavarat, W.: Coupled fixed point theorems in cone metric spaces with a c-distance and applications. Fixed Point Theory Appl. 2012, Article ID 194 (2012)

20. Agarwal, R.P., Sintunavarat, W., Kumam, P.: Coupled coincidence point and common coupled fixed point theorems lacking the mixed monotone property. Fixed Point Theory Appl. 2013, Article ID 22 (2013)

21. Radenović, S.: Remarks on some recent coupled coincidence point results in symmetric G-metric spaces. J. Oper. 2013, Article ID 290525 (2013)

22. Radenović, S.: Remarks on some coupled coincidence point results in partially ordered metric spaces. Arab J. Math. Sci. 20(1), 29-39 (2014)

23. Radenović, S.: Remarks on some coupled fixed point results in partial metric spaces. Nonlinear Funct. Anal. Appl. 18(1), 39-50 (2013)

24. Radenović, S.: Some coupled coincidence points results of monotone mappings in partially ordered metric spaces. Int. J. Anal. Appl. 5(2), 174-184 (2014)

25. Radenović, S.: Coupled fixed point theorems for monotone mappings in partially ordered metric spaces. Kragujev. J. Math. 38(2), 249-257 (2014)

26. Aydi, H., Karapinar, E., Shatanwai, W.: Coupled fixed point results for $(\psi, \varphi)$-weakly contractive condition in ordered partial metric spaces. Comput. Math. Appl. 62, 4449-4460 (2011)

27. Ali, A., Isik, H., Aydi, H., Ameer, E., Lee, J.R., Arshad, M.: On multivalued Suzuki-type $\theta$-contractions and related applications. Open Math. 18, 386-399 (2020)

28. Berinde, V., Borcut, M.: Tripled fixed point theorems for contractive type mappings in partially ordered metric spaces. Nonlinear Anal. 74, 4889-4897 (2011)

29. Borcut, M.: Tripled coincidence theorems for contractive type mappings in partially ordered metric spaces. Appl. Math. Comput. 218, 7339-7346 (2012)

30. Borcut, M., Berinde, V.: Tripled coincidence theorems for contractive type mappings in partially ordered metric spaces. Appl. Math. Comput. 218, 5929-5936 (2012)

31. Choudhury, B.S., Karapınar, E., Kundu, A.: Tripled coincidence point theorems for nonlinear contractions in partially ordered metric spaces. Int. J. Math. Math. Sci. 2012, Article ID 329298 (2012)

32. Aydi, H., Abbas, M., Sintunavarat, W., Kumam, P.: Tripled fixed point of $W$-compatible mappings in abstract metric spaces. Fixed Point Theory Appl. 2012, Article ID 134 (2012)

33. Mustafa, Z., Roshan, J.R., Parvaneh, V.: Existence of a tripled coincidence point in ordered $G_{b}$-metric spaces and applications to a system of integral equations. J. Inequal. Appl. 2013, 453 (2013)

34. Radenović, S.: A note on tripled coincidence and tripled common fixed point theorems in partially ordered metric spaces. Apl. Math. Comput. 236, 367-372 (2014)

35. Ćojbašić Rajić, V., Radenović, S.: A note on tripled fixed point of $\mathbf{W}$-compatible mappings in TVS-cone metric spaces. Thai J. Math. 12(3), 717-728 (2014)

36. Aydi, H., Karapinar, E., Postolache, M.: Tripled coincidence point theorems for weak $\varphi$-contractions in partially ordered metric spaces. Fixed Point Theory Appl. 2012, 44 (2012)

37. Matthews, S.G.: Partial metric topology. In: Proc. 8th Summer Conference on General Topology and Applications. Ann New York Acad. Sci., vol. 728, pp. 183-197 (1994)

38. Aage, C.T., Salunke, J.N.: The results on fixed points in dislocated and dislocated quasi-metric space. Appl. Math. Sci. 2(59), 2941-2948 (2008)

39. Amini-Harandi, A.: Metric-like spaces, partial metric spaces and fixed points. Fixed Point Theory Appl. 2012, 204 (2012)

40. Karapinar, E., Salimi, P.: Dislocated metric space to metric spaces with some fixed point theorems. Fixed Point Theory Appl. 2013, $222(2013)$

41. Al-Mezel, S.A., Chen, C.M., Karapınar, E., Rakocevič, V.: Fixed point results for various $\alpha$-admissible contractive mappings on metric-like spaces. Abstr. Appl. Anal. 2014, Article ID 379358 (2014)

42. Alsulami, H.H., Karapınar, E., Piri, H.: Fixed points of modified F-contractive mappings in complete metric-like spaces. J. Funct. Spaces 2015, Article ID 270971 (2015)

43. Daheriya, R.D., Jain, R., Ughade, M.: Some fixed point theorem for expansive type mapping in dislocated metric space. ISRN Math. Anal. 2012, Article ID 376832 (2012)

44. George, R., Rajagopalan, R., Vinayagam, S.: Cyclic contractions and fixed points in dislocated metric spaces. Int. J. Math. Anal. 9, 403-411 (2013)

45. Hammad, H.A., De la Sen, M.: Solution of nonlinear integral equation via fixed point of cyclic $\alpha_{\llcorner}^{\psi}$-rational contraction mappings in metric-like spaces. Bull. Braz. Math. Soc. 51, 81-105 (2020)

46. Kumari, P.S.: Some fixed point theorems in generalized dislocated metric spaces. Math. Theory Model. 1, 16-22 (2011)

47. Kumari, P.S., Kumar, V.V., Sarma, I.R.: Common fixed point theorems on weakly compatible maps on dislocated metric spaces. Math. Sci. 6, 71 (2012)

48. Aydi, H., Felhi, A., Karapinar, E., Sahmim, S.: A Nadler-type fixed point theorem in dislocated spaces and applications. Miskolc Math. Notes 19(1), 111-124 (2018)

49. Hammad, H.A., Albaqeri, D.M., Rashwan, R.A.: Coupled coincidence point technique and its application for solving nonlinear integral equations in RPOCbML spaces. J. Egypt. Math. Soc. 28, 8 (2020) https://doi.org/10.1186/s42787-019-0064-3

50. Ansari, A.H., Chandok, S., lonescu, C.: Fixed point theorems on $b$-metric spaces for weak contractions with auxiliary functions. J. Inequal. Appl. 2014, 429 (2014)

51. Nashine, H.K., Samet, B.: Fixed point results for mappings satisfying $(\psi, \phi)$-weakly contractive condition in partially ordered metric spaces. Nonlinear Anal. 74, 2201-2209 (2011) 